TRANSACTIONS OF THE

AMERICAN MATHEMATICAL SOCIETY

Volume 353, Number 1, Pages 133-156

S 0002-9947(00)02536-8

Article electronically published on August 3, 2000

\title{
EXTENSION THEORY OF SEPARABLE METRIZABLE SPACES WITH APPLICATIONS TO DIMENSION THEORY
}

\author{
ALEXANDER DRANISHNIKOV AND JERZY DYDAK
}

\begin{abstract}
The paper deals with generalizing several theorems of the covering dimension theory to the extension theory of separable metrizable spaces. Here are some of the main results:

Generalized Eilenberg-Borsuk Theorem. Let $L$ be a countable $C W$ complex. If $X$ is a separable metrizable space and $K * L$ is an absolute extensor of $X$ for some $C W$ complex $K$, then for any map $f: A \rightarrow K, A$ closed in $X$, there is an extension $f^{\prime}: U \rightarrow K$ of $f$ over an open set $U$ such that $L \in A E(X-U)$.

Theorem. Let $K, L$ be countable $C W$ complexes. If $X$ is a separable metrizable space and $K * L$ is an absolute extensor of $X$, then there is a subset $Y$ of $X$ such that $K \in A E(Y)$ and $L \in A E(X-Y)$.

Theorem. Suppose $G_{i}, \ldots, G_{n}$ are countable, non-trivial, abelian groups and $k>0$. For any separable metrizable space $X$ of finite dimension $\operatorname{dim} X>0$, there is a closed subset $Y$ of $X$ with $\operatorname{dim}_{G_{i}} Y=\max \left(\operatorname{dim}_{G_{i}} X-k, 1\right)$ for $i=1, \ldots, n$.

Theorem. Suppose $W$ is a separable metrizable space of finite dimension and $Y$ is a compactum of finite dimension. Then, for any $k, 0<k<\operatorname{dim} W-$ $\operatorname{dim} Y$, there is a closed subset $T$ of $W$ such that $\operatorname{dim} T=\operatorname{dim} W-k$ and $\operatorname{dim}(T \times Y)=\operatorname{dim}(W \times Y)-k$.

Theorem. Suppose $X$ is a metrizable space of finite dimension and $Y$ is a compactum of finite dimension. If $K \in A E(X)$ and $L \in A E(Y)$ are connected $C W$ complexes, then $K \wedge L \in A E(X \times Y)$.
\end{abstract}

\section{INTRODUCTION}

Recall that the cohomological dimension $\operatorname{dim}_{G} X$ is the smallest integer $n$ such that $\check{H}^{n+1}(X, A ; G)=0$ for all closed subsets $A$ of $X$.

Ever since the introduction of the cohomological dimension by P.S.Alexandroff [A], it was clear that there is a similarity between dimension and cohomological dimension. However, until recently, the dimension was investigated using mostly settheoretic methods, and the cohomological dimension was investigated using mostly algebraic methods. Let us recall the basic results of the covering dimension theory En:

Received by the editors July 14, 1995 and, in revised form, February 5, 1999.

1991 Mathematics Subject Classification. Primary 55M10, 54F45.

Key words and phrases. Dimension, cohomological dimension, ANR's, absolute extensors.

The first and second authors were supported in part by grants DMS-9696238 and DMS-9704372, respectively, from the National Science Foundation. 
Basic results of dimension theory of separable metric spaces.

Theorem 1.1 (Hurewicz-Wallman [En], p. 90). $\operatorname{dim} X \leq n$ iff $S^{n} \in A E(X)$, i.e. any map $f: A \rightarrow S^{n}$ extends over $X$ if $A$ closed in $X$.

Theorem 1.2 (Urysohn-Menger [En], p. 45). $\operatorname{dim}(A \cup B) \leq \operatorname{dim} A+\operatorname{dim} B+1$.

Theorem 1.3 (Urysohn-Tumarkin-Hurewicz [En], p. 45). If $\operatorname{dim} X=m+n+1$, then there are subsets $A$ and $B$ of $X$ such that $X=A \cup B$ and $\operatorname{dim} A \leq m$, $\operatorname{dim} B \leq n$.

Theorem 1.4 (Tumarkin En, p. 45). If $\operatorname{dim} X=n$, then there is a completely metrizable space $X^{\prime}$ containing $X$ and with $\operatorname{dim} X^{\prime}=n$.

Theorem 1.5 (Hurewicz [En], p. 61). If $\operatorname{dim} X=n$, then there is a compact metrizable space $X^{\prime}$ containing $X$ and with $\operatorname{dim} X^{\prime}=n$.

Theorem 1.6 (Menger [En], p. 46). $\operatorname{dim}(X \times Y) \leq \operatorname{dim} X+\operatorname{dim} Y$.

Theorem 1.7 (Nobeling [En, p. 120). There is a universal separable metrizable space of a given dimension.

Theorem 1.8 (Lefschetz [En], p. 126). There is a universal compact metrizable space of a given dimension.

Since $\operatorname{dim}_{\mathbf{Z}} X \leq n$ can be characterized as $K(\mathbf{Z}, n) \in A E(X)$, it was natural to seek analogs of the above statements for the cohomological dimension. So far, only the existence of universal compact spaces of a given integral cohomological dimension has eluded researchers in the field. Here are the analogs of 1.1-1.8 for the integral cohomological dimension:

Main results of integral dimension theory of separable metric spaces.

Theorem 1.9 (Cohen $\left[\mathrm{Co}\right.$ ). $\operatorname{dim}_{\mathbf{Z}} X \leq n$ iff $K(\mathbf{Z}, n) \in A E(X)$, i.e., any map $f: A \rightarrow K(\mathbf{Z}, n)$ extends over $X$ if $A$ closed in $X$.

Theorem 1.10 (Rubin $[\mathrm{Ru}]) \cdot \operatorname{dim}_{\mathbf{Z}}(A \cup B) \leq \operatorname{dim}_{\mathbf{Z}} A+\operatorname{dim}_{\mathbf{Z}} B+1$.

Theorem 1.11 (Dranishnikov Dra $)$. There is a compactum $X$ such that $\operatorname{dim}_{\mathbf{Z}} X$ $=3$, and $X \neq A \cup B$ with $\operatorname{dim}_{\mathbf{Z}} A \leq 1, \operatorname{dim}_{\mathbf{Z}} B \leq 1$

Theorem 1.12 (Rubin-Schapiro [R-S] ). If $\operatorname{dim}_{\mathbf{Z}} X=n$, then there is a completely metrizable space $X^{\prime}$ containing $X$ and with $\operatorname{dim}_{\mathbf{Z}} X^{\prime}=n$.

Theorem 1.13 (Dydak-Walsh $\left[\mathrm{D}^{\prime} \mathrm{W}_{1}\right)$. There is a separable metrizable space $X$ such that $\operatorname{dim}_{\mathbf{Z}} X=4$ and $\operatorname{dim}_{\mathbf{Z}} X^{\prime}>4$ for any compact space $X^{\prime}$ containing $X$.

Theorem 1.14 (Rubin-Schapiro [R-S]). $\operatorname{dim}_{\mathbf{Z}}(X \times Y) \leq \operatorname{dim}_{\mathbf{Z}} X+\operatorname{dim}_{\mathbf{Z}} Y$.

Theorem 1.15 (Dydak-Mogilski $[\mathrm{D}-\mathrm{M}]$ ). There is a universal separable metrizable space of a given integral cohomological dimension.

Question 1.16. Is there is a universal compact metrizable space of a given integral cohomological dimension?

With the exception of 1.9 , all of the above results were proved with difficulty exceeding similar results for the covering dimension. Especially interesting is the history of generalizing the Urysohn-Menger Theorem (see $\left.[\mathrm{Ru}], \overline{\mathrm{D}-\mathrm{W}_{2}}\right], \mathrm{D}-\mathrm{R}-\mathrm{S}_{1}$, D-R,,$\overline{\mathrm{Dy}_{2}}$ and $\left.\overline{\mathrm{Dy}} \mathbf{3}\right)$. 
In 1992, during a workshop on cohomological dimension theory in Knoxville,TN, A.Dranishnikov presented certain results (see Dra 4 ) of extraordinary dimension theory, which he called "Extension Theory". Roughly speaking, one can create a new equivalence of compacta: $X \sim Y$ iff $K \in A E(X)$ is equivalent to $K \in A E(Y)$ for any CW complex $K$. Dually, one can introduce a new equivalence of $\mathrm{CW}$ complexes: $K \sim L$ iff $K \in A E(X)$ is equivalent to $L \in A E(X)$ for any compactum $X$. Dranishnikov's idea was that Extension Theory ought to investigate these equivalences. Inspired by his talk, J.Dydak thought that perhaps Extension Theory could be a theory encompassing both the covering and the cohomological dimension theories. Since at that time J.Dydak was working on Kuzminov's question "Does $\operatorname{dim}_{G}(A \cup B) \leq \operatorname{dim}_{G} A+\operatorname{dim}_{G} B+1$ hold ?", it was natural to try to translate:

$$
\operatorname{dim}(A \cup B) \leq \operatorname{dim} A+\operatorname{dim} B+1
$$

into the language of Extension Theory. A simple translation is:

$$
\text { if } S^{m} \in A E(A) \text { and } S^{n} \in A E(B) \text {, then } S^{m+n+1} \in A E(A \cup B) \text {. }
$$

What is the connection between $S^{m}, S^{n}$, and $S^{m+n+1}$ ?

The answer is: $S^{m+n+1}$ is the join of $S^{m}$ and $S^{n}$.

Using this observation, J.Dydak $\mathrm{Dy}_{2}$ proposed the following (either as specific conjectures or problems which subsequently were converted into conjectures in $[\bar{D}-\mathrm{M}]$ ):

\section{Candidates for basic results of Extension Theory of separable metric} spaces.

Conjecture 1.17. If $K \in A E(A)$ and $L \in A E(B)$ are $C W$ complexes, then $K * L \in$ $A E(A \cup B)$.

Conjecture 1.18. If $K * L \in A E(X)$, then there are subsets $A, B$ of $X$ such that $X=A \cup B, K \in A E(A)$, and $L \in A E(B)$.

Conjecture 1.19. If $K \in A E(X)$ is a countable $C W$ complex, then there is a completion $\bar{X}$ of $X$ with $K \in A E(\bar{X})$.

Conjecture 1.20. Let $K$ be a countable $C W$ complex. Any $X$ with $K \in A E(X)$ admits a compactification $\bar{X}$ with $K \in A E(\bar{X})$ iff $K$ is homotopy dominated by a finite polyhedron.

Conjecture 1.21. If $K, L$ are $C W$ complexes and $K \in A E(X), L \in A E(Y)$, then $K \wedge L \in A E(X \times Y)$.

Conjecture 1.22. Suppose $K$ is a countable $C W$ complex. There is a universal separable space in the class $\{X \mid K \in A E(X)\}$.

Conjecture 1.23. Suppose $K$ is a $C W$ complex. The class $\{X \mid K \in A E(X)\}$ has a universal compact space iff $K$ is homotopy dominated by a finite polyhedron.

Several of the conjectures above were subsequently answered positively:

a. Conjecture 1.17 by J.Dydak $\overline{\mathrm{Dy}_{2}}$ for arbitrary metrizable spaces $A, B$ and arbitrary CW complexes $K, L$.

b. Conjecture 1.18 by A.Dranishnikov $\mathrm{Dra}_{2}$ for compact spaces $X$.

c. Conjecture 1.19 by W.Olszewski $\mathrm{Ol}_{1}$ ].

d. Conjecture 1.21 by A.Dranishnikov Dras in the special case $Y=[0,1]$ and $L=S^{1}$. 
e. Conjecture 1.22 by W.Olszewski $\left[\mathrm{Ol}_{2}\right]$. See also related work by M.Zarichnyi [Z].

Not only do the results of Extension Theory have simpler proofs than their predecessors in cohomological dimension theory; they give an explanation of certain phenomena in cohomological dimension theory and, sometimes, are more general than expected. Let us demonstrate this in the case of Dranishnikov's Dra 2 result:

Theorem 1.24. Suppose $X$ is a compactum and $K, L$ are countable $C W$ complexes. If $K * L \in A E(X)$, then there are subsets $A, B$ of $X$ such that $X=A \cup B$, $K \in A E(A)$, and $L \in A E(B)$.

He applied it successfully to the Mapping Intersection Problem in codimensions different from two. It turns out that Theorem 1.24 also implies Dranishnikov's Realization Theorem [Dra 1 which gives necessary and sufficient conditions for existence of compacta with prescribed values of cohomological dimension for each basic group. In the process of proving Theorem 1.24, Dranishnikov generalized a useful result from classical dimension theory:

Theorem 1.25 (Eilenberg-Borsuk Ei], Bor). If $X$ is a separable metrizable space of dimension $n$, then for any map $f: A \rightarrow S^{k}, A$ closed in $X$ and $k<n$, there is an extension $f^{\prime}: U \rightarrow K$ of $f$ over an open set $U$ such that $\operatorname{dim}(X-U) \leq n-k-1$.

Here is that generalization:

Theorem 1.26 (A.Dranishnikov $\left.\mathrm{Dra}_{2}\right)$ ). Let $K, L$ be countable $C W$ complexes. If $X$ is a compactum and $K * L$ is an absolute extensor of $X$, then for any map $f: A \rightarrow K, A$ closed in $X$, there is an extension $f^{\prime}: U \rightarrow K$ of $f$ over an open set $U$ such that $L \in A E(X-U)$.

The purpose of this paper is to extend results of the Extension Theory of compacta to separable metrizable spaces, to generalize some results of dimension theory, and to provide a partial answer to Conjecture 1.21. In particular, we will generalize the following well-known result:

Theorem 1.27 (En, p. 41). If $X$ is a separable metrizable space of finite dimension $\operatorname{dim} X=n$, then for all $k<n$ there is a closed subset $Y$ of $X$ of dimension $k$.

One may say that instead of studying extension types as in Dra7, we are trying to see if, given $K \in A E(X), L \in A E(Y)$, and given a binary operation $R(\cdot, \cdot)$ on metrizable spaces (for example, $R(X, Y)=X \cup Y$ or $R(X, Y)=X \times Y$ ), one can match $R$ with a binary operation $S$ on CW complexes so that $S(K, L) \in$ $A E(R(X, Y))$.

When translating results from Extension Theory to Cohomological Dimension Theory we find that the following theorem is of fundamental importance:

Theorem 1.28. Suppose $X$ is a metrizable space and $K$ is a connected $C W$ complex. Consider the following conditions:

1. $K \in A E(X)$.

2. $S P^{\infty}(K) \in A E(X)\left(S P^{\infty}(K)\right.$ is the infinite symmetric product of $K$-see [D-T]).

3. $\operatorname{dim}_{H_{m}(K ; \mathbf{Z})} X \leq m$ for all $m \geq 0$.

4. $\operatorname{dim}_{\pi_{m}(K ; \mathbf{Z})} X \leq m$ for all $m \geq 0$. 
Then, Condition 1) implies Condition 2). If $K$ is simply connected, then Conditions 2-3-4 are equivalent. If $X$ is of finite dimension and $K$ is simply connected, then Conditions 1-2-3-4 are equivalent.

For $X$ compact, Theorem 1.28 is due to A.Dranishnikov $\mathrm{Dra}_{3}$. Subsequently, it has been generalized to metrizable spaces by J.Dydak $\mathrm{Dy}_{1}$, $\mathrm{Dy}_{2}$.

\section{Preliminary Results}

We need the following improvement of Tietze Extension Theorem:

Lemma 2.0. Suppose $f: A \rightarrow[0,1]$ is continuous and $A$ is a closed subset of a metrizable space $X$. There is an extension $F: X \rightarrow[0,1]$ of $f$ such that $F^{-1}(0)=$ $f^{-1}(0)$ and $F^{-1}(1)=f^{-1}(1)$.

Proof. Choose a decreasing sequence of open neighborhoods $U_{n}$ of $f^{-1}(0)$ in $X$ whose intersection is $f^{-1}(0)$. Choose a decreasing sequence of open neighborhoods $V_{n}$ of $f^{-1}(1)$ in $X$ whose intersection is $f^{-1}(1)$. Given $n$, extend $f \mid A-\left(U_{n} \cup V_{n}\right)$ : $A-\left(U_{n} \cup V_{n}\right) \rightarrow(0,1)$ to $g_{n}: X-\left(U_{n} \cup V_{n}\right) \rightarrow(0,1)$. Extend $g_{n} \cup f$ to $f_{n}: X \rightarrow[0,1]$. Now, define $F: X \rightarrow[0,1]$ as $\sum_{n=1}^{\infty} f_{n} / 2^{n}$.

In most of the results of this paper, $K, L$ are CW complexes. Occasionally, however, we will switch to simplicial complexes with the metric topology $K_{m}$. Obviously, $K$ equipped with the CW topology contains more open sets, and therefore it is easier to construct maps into $K_{m}$ rather than into $K$. On the other hand, given a map into a CW complex, one can take advantage of restrictions it imposes on the map (see Parts 6-7 of Lemma 2.1 below) and get more elegant proofs of some results. From the homotopy point of view there is no difference between $K_{m}$ and $K$, and the purpose of the following lemma is to put together various results scattered throughout the literature; results which support this claim.

Lemma 2.1. Suppose $X$ is a metrizable space, and $K$ is either a $C W$ complex or a simplicial complex with the metric topology. Then:

1. $K \in A N E(X)$, i.e., and if $f: A \rightarrow K$ is a map and $A$ is a closed subset of $X$, then there are an open neighborhood $U$ of $A$ in $X$ and a map $F: U \rightarrow K$ so that $F \mid A=f$.

2. (Homotopy Extension Theorem) Any $f: A \times[0,1] \cup X \times\{0\} \rightarrow K$, where $A$ is a closed subset of $X$, extends over $X \times[0,1]$.

3. If $f: A \rightarrow K$ is a map, $A \subset X$, and $\mathcal{U}$ an open cover of a metric simplicial complex $K$, then there are an open neighborhood $U$ of $A$ in $X$, a map $F$ : $U \rightarrow K$ and a homotopy $H: A \times[0,1] \rightarrow K$ joining $f$ and $F \mid A$ such that each path $H(\{x\} \times I)$ of $H$ is contained in an element of $\mathcal{U}$. Moreover, if $B \subset A$ is a closed subset of $X$, then one can construct $H$ so that $H(\{x\} \times I)$ is a one-point set for all $x \in B$.

4. $K$ is homotopy equivalent to a simplicial complex equipped with the metric topology.

5. If $K$ is a simplicial complex, then the identity map $i: K \rightarrow K_{m}$ is a homotopy equivalence.

6. If $K$ is a $C W$ complex, then for any map $f: X \rightarrow K$ and for any $x \in X$ there is a neighborhood $U$ of $x$ such that $f(U)$ is contained in a finite subcomplex of $K$. 
7. If $K$ is a $C W$ complex and if $X$ is separable, then for any map $f: X \rightarrow K$. $f(X)$ is contained in a countable subcomplex of $K$.

8. If $K$ is a metric simplicial complex, then $f: X \rightarrow K$ is continuous iff $\phi_{v} f$ is continuous for all vertices $v$ of $K\left(\left\{\phi_{v}(x)\right\}\right.$ are barycentric coordinates of $\left.x=\sum_{v \in K^{(0)}} \phi_{v}(x) \cdot v\right)$.

Proof. 1. If $K$ is a CW complex, see [Ko]. If $K$ is a metric simplicial complex, see M-S, Theorem 11 on p.304.

2. See [M-S], Theorem 9 on p.41.

3. If $K$ is a simplicial complex with the metric topology, one can use [M-S], Corollary 3 and Remark 3 on p.81.

4. See [M-S], Theorem 5 on p.317.

5. See [M-S], Theorem 10 on p.302.

6. Assume that $f(V)$ is not contained in a finite subcomplex of $K$ for any neighborhood $V$ of $x$ in $X$. For each $n$ choose, by induction, $x_{n}$ so that $0<$ $\operatorname{dist}\left(x, x_{n}\right)<1 / n$ and $f\left(x_{n}\right)$ does not belong to the smallest subcomplex of $K$ containing $f(x)$ and all $f\left(x_{k}\right), k<n$. The set $S=\left\{f\left(x_{n}\right)\right\}_{n>0}$ is a closed subset of $K$, which contradicts continuity of $f$.

7. Each $x \in X$ has a neighborhood $V$ such that $f(V)$ is contained in a finite subcomplex of $K$. Now, $X$ can be covered by a countable family of $V_{i}$ such that each $f\left(V_{i}\right)$ is contained in a countable subcomplex of $K$.

8. See [M-S], Theorem 8 on p.301.

Definition 2.2. Given two CW complexes $K$ and $L$, their join $K * L$ is formed as the adjoining space $K \oplus L \cup_{p r} K \times L \times[0,1]$, where $p r: K \times L \times\{0,1\} \rightarrow K \oplus L$ is defined by

$$
\operatorname{pr}(a, b, t)= \begin{cases}a & \text { if } t=0, \\ b & \text { if } t=1 .\end{cases}
$$

Notice that $K * L$ is homeomorphic to the union $M\left(\pi_{1}\right) \cup M\left(\pi_{2}\right)$ of mapping cylinders of projections $\pi_{1}: K \times L \rightarrow K$ and $\pi_{2}: K \times L \rightarrow L$, where we assume $M\left(\pi_{1}\right) \cap$ $M\left(\pi_{2}\right)=K \times L$. Also, it is clear that this definition can be used to define the join of any two topological spaces.

Notice that there are canonical projections $\pi: K * L \rightarrow[0,1], \pi_{K}: K * L-L \rightarrow K$ and $\pi_{L}: K * L-K \rightarrow L$.

Definition 2.3. Given two simplicial complexes $K$ and $L$, their simplicial join $K * L$ is formed by declaring $\left\langle s_{0}, \ldots, s_{k}, t_{0}, \ldots, t_{l}\right\rangle$ to be its simplex iff $\left\langle s_{0}, \ldots, s_{k}\right\rangle$ is a simplex in $K$ and $\left\langle t_{0}, \ldots, t_{l}\right\rangle$ is a simplex in $L$. It carries a natural CW structure; its cells are of the form $s * \sigma$, where $s$ is a simplex of $K$ and $\sigma$ is a simplex of $L$. Notice that $K * L$ with the CW topology is homeomorphic to the union $M\left(\pi_{K}\right) \cup M\left(\pi_{L}\right)$ of mapping cylinders of projections $\pi_{K}: K \times L \rightarrow K$ and $\pi_{L}: K \times L \rightarrow L$, where we assume $M\left(\pi_{K}\right) \cap M\left(\pi_{L}\right)=K \times L$. Thus, the simplicial join is related to the abstract join. As in the case of the abstract join, there are canonical projections $\pi: K * L \rightarrow[0,1], \pi_{K}: K * L-L \rightarrow K$ and $\pi_{L}: K * L-K \rightarrow L$. Namely, any point $x \in K * L$ can be expressed as $t \cdot u+(1-t) \cdot v$, where $t \in[0,1], u \in K$, and $v \in L$. $t$ is unique, and we put $\pi(x)=t$. $u$ is unique if $x \notin L$, and we put $\pi_{K}(x)=u$. Similarly, $v$ is unique if $x \notin K$, and we put $\pi_{L}(x)=v$.

The following lemma is crucial in all constructions involving maps to joins of metric simplicial complexes. 
Lemma 2.4. Suppose $K, L$ are metric simplicial complexes and let $\pi: K * L \rightarrow$ $[0,1], \pi_{K}: K * L-L \rightarrow K$, and $\pi_{L}: K * L-K \rightarrow L$ be canonical projections. If $X$ is a metrizable space, then there is a one-to-one correspondence between maps $f: X \rightarrow K * L$ and 5-tuples $(U, V, g: U \rightarrow K, h: V \rightarrow L, \alpha: X \rightarrow[0,1])$ satisfying the following conditions:

1. $X=U \cup V, U$ and $V$ open in $X$;

2. $\alpha^{-1}[0,1)=U$ and $\alpha^{-1}(0,1]=V$.

Namely, given $f: X \rightarrow K * L$, define $U=f^{-1}(K * L-L), V=f^{-1}(K * L-K)$, $g=\pi_{K} \circ f, h=\pi_{L} \circ f$ and $\alpha=\pi \circ f$. Conversely, given $(U, V, g: U \rightarrow K, h: V \rightarrow$ $L, \alpha: X \rightarrow[0,1])$, one defines $f$ as follows:

$$
f(x)= \begin{cases}g(x) & \text { if } x \in U-V, \\ (1-\alpha(x)) \cdot g(x)+\alpha(x) \cdot h(x) & \text { if } x \in U \cap V, \\ h(x) & \text { if } x \in V-U .\end{cases}
$$

The map $f$ defined as above will be denoted by $g *_{\alpha} h$.

Proof. The only thing we need to verify is that $f \mid U \cup V$ is continuous. Notice that each point $x$ of a simplicial complex $M$ can be uniquely written as

$$
x=\sum_{v \in M^{(0)}} \phi_{v}(x) \cdot v,
$$

where $M^{(0)}$ is the set of vertices of $M\left(\left\{\phi_{v}(x)\right\}\right.$ are called barycentric coordinates of $x$ ). To prove the continuity of $f$ we need to show that $\phi_{v} f$ is continuous for all vertices $v$ of $K * L$ (see $\mathrm{M}-\mathrm{S}$, Theorem 8 on p.301, or Part 8 of Lemma 2.1). Without loss of generality we may assume that $v \in K^{(0)}$. Then,

$$
\phi_{v} f(x)=(1-\alpha(x)) \cdot \phi_{v} g(x) \text { for all } x \in U
$$

and

$$
\phi_{v} f(x)=0 \text { for all } x \in U-V .
$$

Clearly, $\phi_{v} f \mid U$ is continuous. If $x_{0} \in V-U$ and $x_{n} \rightarrow x_{0}, x_{n} \in U$, then $\alpha\left(x_{n}\right) \rightarrow 1$ and $0 \leq \phi_{v} g\left(x_{n}\right) \leq 1$. Consequently, $\phi_{v} f\left(x_{n}\right) \rightarrow 0=\phi_{v} f\left(x_{0}\right)$.

Lemma 2.5. Suppose $K, L$ are metric simplicial complexes, and $Y$ is a metrizable space such that $K * L \in A E(X)$ for a subset $X$ of $Y$. Given closed subsets $A$ of $X$ and $B$ of $Y$, and given maps $f: A \rightarrow K, g: B \rightarrow L$, there are a neighborhood $U$ of $A \cup X$ in $Y$ and a map $F: U \rightarrow K * L$ such that $F(A) \subset K * L-L$, $F(B) \subset K * L-K, F \circ \pi_{K} \mid A=f$ and $F \circ \pi_{L} \mid B \simeq g$, where $\pi_{K}: K * L-L \rightarrow K$ and $\pi_{L}: K * L-K \rightarrow L$ are projections.

Proof. Extend $f$ up to homotopy to $f^{\prime}: V \rightarrow K$, and extend $g$ up to homotopy to $g^{\prime}: W \rightarrow L$ for some neighborhood $V$ of $A$ in $Y$ and some neighborhood $W$ of $B$ in $Y$ (see Part 3 of Lemma 2.1). Choose a map $\alpha: V \cup W \rightarrow[0,1]$ such that $\alpha^{-1}(0)=V-W$ and $\alpha^{-1}(1)=W-V$. Now, use Lemma 2.4 and produce $G: V \cup W \rightarrow K * L$. Since $X \cap(A \cup B)$ is a closed subset of $X$, there is an extension $H: A \cup X \rightarrow K * L$ of $G \mid X \cap(A \cup B)$. Let $\mathcal{U}=\{K * L-L, K * L-K\}$ be the open cover of $K * L$. Using Part 3 of Lemma 2.1, we can find an open neighborhood $U$ of $A \cup X$ and an extension $F: U \rightarrow K * L$ of $F \mid A$, such that $F \mid A \cup X$ is homotopic to $H$ in such a way that the paths of homotopy are contained in $\mathcal{U}$. It is clear that $F$ is the required map. 
The following lemma is useful when discussing generalizations of the EilenbergBorsuk Theorem.

Lemma 2.6. Suppose $K, L$ are metric simplicial complexes and $X$ is a metrizable space. Let $v$ be the vertex of the cone Cone $(K)$ of $K$. The following conditions are equivalent:

1. For any map $f: A \rightarrow K, A$ closed in $Y \subset X$, there is an extension $f^{\prime}: U \rightarrow K$ of $f$ over an open set $U$ of $Y$ such that $L \in A E(Y-U)$.

2. For any map $f: A \rightarrow K, A$ closed in $Y \subset X$, there is an extension $F: Y \rightarrow$ Cone $(K)$ of $A \rightarrow K \hookrightarrow$ Cone $(K)$ such that $L \in A E\left(F^{-1}(v)\right)$.

3. For any map $f: A \rightarrow$ Cone $(K)$, A closed in $Y \subset X$, there is an extension $F: Y \rightarrow$ Cone $(K)$ of $f$ such that $L \in A E\left(F^{-1}(v)-A\right)$.

Proof. Let $\pi_{K}:$ Cone $(K)-\{v\} \rightarrow K$ and $\pi:$ Cone $(K) \rightarrow[0,1]$ be projections.

$1 \rightarrow 2$. Notice that $\operatorname{Cone}(K)$ is homeomorphic to the join $K * v$. Take an extension $f^{\prime}: U \rightarrow K$ of $f$ over an open set $U$ such that $L \in A E(Y-U)$. Choose a map $\alpha: Y \rightarrow[0,1]$ such that $\alpha^{-1}(0)=A$ and $Y-U=\alpha^{-1}(1)$. Put $V=\alpha^{-1}(0,1]$, $g^{\prime}: V \rightarrow\{v\}$, and use Lemma 2.4.

$2 \rightarrow 3$. Choose an extension $g: U \rightarrow K$ of $\pi_{K} \circ f: A-f^{-1}(v) \rightarrow K$ such that $L \in A E\left(Y-f^{-1}(v)-U\right)$ and $U$ is open in $Y-f^{-1}(v)$. According to Lemma 2.0 there is an extension $H: Y \rightarrow[0,1]$ of $h=\pi \circ f$ so that $H^{-1}(1)=Y-U$. Finally, use Lemma 2.4, or define $F: Y \rightarrow \operatorname{Cone}(K)$ by

$$
F(x)= \begin{cases}{[g(x), H(x)]} & \text { if } x \in U, \\ v & \text { if } x \in Y-U .\end{cases}
$$

$3 \rightarrow 1$. Extend $f: A \rightarrow \operatorname{Cone}(K)$ to $F: Y \rightarrow \operatorname{Cone}(K)$ so that $L \in$ $A E\left(F^{-1}(v)-A\right)$. Notice that $F^{-1}(v)-A=F^{-1}(v)$ and put $U=Y-F^{-1}(v)$, $f^{\prime}=\pi_{K} \circ F: U \rightarrow K$.

Theorem 2.7. Let $K, L$ be $C W$ complexes, and let $X$ be a metrizable space. If, for any map $f: A \rightarrow K, A \subset X$, there is an extension $f^{\prime}: U \rightarrow K$ of $f$ over an open set $U$ of $Y=X-\left(\operatorname{cl}_{X}(A)-A\right)$ such that $L \in A E(Y-U)$, then $K * L$ is an absolute extensor of $X$.

Proof. Since, by Part 2 of Lemma 2.1, being an absolute extensor is a property of the homotopy type, we may assume that both $K$ and $L$ are metric simplicial complexes. Suppose $C$ is a closed subset of $X$, and $g: C \rightarrow K * L$ is a map. Let $A=C-g^{-1}(L)$, and let $f: A \rightarrow K$ be defined by $f(x)=\pi_{K}(g(x))$ for $x \in A$. Choose an extension $f^{\prime}: U \rightarrow K$ of $f$ over a neighborhood $U$ of $A$ in $Y=X-\left(\operatorname{cl}_{X}(A)-A\right)$ so that $L \in A E(Y-U)$. Notice that $(Y-U) \cup g^{-1}(L)$ is closed in $X$, and the map $\pi_{L} \circ g: C-g^{-1}(K) \rightarrow L$ can be extended to $h:(Y-U) \cup C-g^{-1}(K) \rightarrow L$. Now, extend $h$ to $h^{\prime}: V \rightarrow L$ for some neighborhood $V$ of $(Y-U) \cup C-g^{-1}(K)$ in $X-g^{-1}(K)$. Let $\pi: K * L \rightarrow[0,1]$ be the projection. Extend the composition $\pi \circ g$ over $(X-U) \cup(X-V) \cup C$ by sending $X-U$ to 1 , and by sending $X-V$ to 0 . Extend the resulting map to $\alpha: X \rightarrow[0,1]$ so that $\alpha^{-1}(0)=X-V$ and $\alpha^{-1}(1)=X-U$ (see Lemma 2.0). Now, use Lemma 2.4.

\section{Main Results}

The following result is fundamental to section 3 of the paper. We arrived at it after analyzing Olszewski's paper $\mathrm{Ol}_{1}$, and we believe it offers a significant 
simplification of his proof (see Theorem 3.6 in the present paper). To simplify its formulation we need the concept of a $\cup \cap$-closed basis:

Definition 3.1. A family $\mathcal{C}$ of closed subsets of the Hilbert cube $Q$ is called a $\cup \cap$-closed basis provided the family $\left\{\operatorname{Int}_{Q} D \mid D \in \mathcal{C}\right\}$ is a basis of $Q, \mathcal{C}$ is closed under finite unions and finite intersections, and $Q-\operatorname{Int}_{Q} A \in \mathcal{C}$ if $A \in \mathcal{C}$.

Theorem 3.2. Let $K$ be a $C W$ complex and let $\mathcal{C}$ be a $\cup \cap$-closed basis of $Q$. Suppose $\mathcal{F}$ is a non-empty family of triples $(f, S, D)$ such that $S \subset Q, D \in \mathcal{C}$, and $f: S \cup D \rightarrow K$ is a map. Let $X=\bigcap_{(f, S, D) \in \mathcal{F}} S . K \in A E(X)$ if the following two conditions are satisfied:

1. $\left(f \mid\left(S^{\prime} \cap S\right) \cup C, S^{\prime} \cap S, C\right) \in \mathcal{F}$ if $(f, S, D) \in \mathcal{F},\left(f^{\prime}, S^{\prime}, D^{\prime}\right) \in \mathcal{F}$, and $C \subset$ $D^{\prime} \cap D, C \in \mathcal{C}$.

2. Given $(f, S, D) \in \mathcal{F}$ and given an extension $g:(S \cap B) \cup D \cup C \rightarrow K$ of $f \mid(S \cap B) \cup D$, where $B, C \in \mathcal{C}$ with $B \cap C=\emptyset$, there is $\left(f^{\prime}, S^{\prime}, D \cup C\right) \in \mathcal{F}$ so that $S^{\prime} \subset S$ and $g\left|\left(S^{\prime} \cap B\right) \cup D \cup C \simeq f^{\prime}\right|\left(S^{\prime} \cap B\right) \cup D \cup C$.

Proof. Define a new family $\mathcal{G}$ of triples $(f, S, D)$ as follows: $(g, S, D) \in \mathcal{G}$ iff $g$ : $S \cup D \rightarrow K$ is a map and there is $(f, S, D) \in \mathcal{F}$ with $f$ homotopic to $g$. $\mathcal{G}$ has stronger properties than $\mathcal{F}$ :

$1^{\prime} .\left(f \mid\left(S^{\prime} \cap S\right) \cup C, S^{\prime} \cap S, C\right) \in \mathcal{G}$ if $(f, S, D) \in \mathcal{G}$ and $\left(f^{\prime}, S^{\prime}, D^{\prime}\right) \in \mathcal{G}$, and $C \subset D^{\prime} \cap D, C \subset \mathcal{C}$.

$2^{\prime}$. Given $(f, S, D) \in \mathcal{G}$ and given an extension $g:(S \cap B) \cup D \cup C \rightarrow K$ of $f \mid(S \cap B) \cup D$, where $B, C \in \mathcal{C}$ with $B \cap C=\emptyset$, there is $\left(f^{\prime}, S^{\prime}, D \cup C\right) \in \mathcal{G}$ so that $S^{\prime} \subset S$ and $g\left|\left(S^{\prime} \cap B\right) \cup D \cup C=f^{\prime}\right|\left(S^{\prime} \cap B\right) \cup D \cup C$.

Property $\left.1^{\prime}\right)$ is obvious. To prove Property $\left.2^{\prime}\right)$, notice that $(S \cap B) \cup D \cup C$ is closed in $S \cup D \cup C$ and $(S \cap B) \cup D$ is closed in $(S \cap B) \cup D \cup C$. Since $(f, S, D) \in \mathcal{G}$, there is $(h, S, D) \in \mathcal{F}$ with $f \simeq h$. Since $g|(S \cap B) \cup D \simeq h|(S \cap B) \cup D$, there is $g^{\prime}:(S \cap B) \cup D \cup C \rightarrow K$ homotopic to $g$ so that $g^{\prime}$ is an extension of $h \mid(S \cap B) \cup D$. Pick $\left(g^{\prime \prime}, S^{\prime}, D \cup C\right) \in \mathcal{F}$ so that $S^{\prime} \subset S$ and $g^{\prime \prime}\left|\left(S^{\prime} \cap B\right) \cup D \cup C \simeq g^{\prime}\right|\left(S^{\prime} \cap B\right) \cup$ $D \cup C$. By the Homotopy Extension Theorem, there is $\left(f^{\prime}, S^{\prime}, D \cup C\right) \in \mathcal{G}$ with $f^{\prime}\left|\left(S^{\prime} \cap B\right) \cup D \cup C=g\right|\left(S^{\prime} \cap B\right) \cup D \cup C$.

To prove 3.2 it suffices to show that any map $g: A \rightarrow K$ extends over $X$ up to homotopy if $A$ is a closed subset of $X$. Since any map $g: A \rightarrow K$ extends up to homotopy over a neighborhood of $A$ in $Q$ (see Part 3 of Lemma 2.1), we need to show that given a closed subset $A$ of $X$, and given a map $g: V \rightarrow K, V$ being a neighborhood of $A$ in $Q$, the map $g \mid A$ extends over $X$. We will construct by induction the following objects:

i. a decreasing sequence $A_{n} \in \mathcal{C}$,

ii. increasing sequences $B_{n} \in \mathcal{C}$ and $D_{n} \in \mathcal{C}$,

iii. elements $\left(g_{n}, S_{n}, A_{n} \cap B_{n}\right) \in \mathcal{G}$,

such that the following conditions are satisfied:

1. $\bigcap_{n=1}^{\infty} A_{n}=\operatorname{cl}_{Q} A$

2. $V=\bigcup_{n=1}^{\infty} B_{n}$

3. $S_{n+1} \subset S_{n}$,

4. $g_{n+1}\left|S_{n+1}-\operatorname{Int}_{Q} A_{n}=g_{n}\right| S_{n+1}-\operatorname{Int}_{Q} A_{n}$,

5. $g_{n}\left|A_{n} \cap B_{n}=g\right| A_{n} \cap B_{n}$. 
Notice that for any closed subset $B$ of $Q$, the family $\{D \in \mathcal{C} \mid B \subset \operatorname{Int} D\}$ is a basis of neighborhoods of $B$. Therefore, there is a sequence $A_{n} \in \mathcal{C}$ with $A_{n+1} \subset \operatorname{Int} A_{n}$ and $\bigcap_{n=1}^{\infty} A_{n}=\operatorname{cl}_{Q} A$. Choose $B \in \mathcal{C}, B \subset V$ with $g \mid B \simeq$ const. Choose a sequence $B_{n} \in \mathcal{C}$ with $B_{n+1} \supset \operatorname{Int} B_{n}, B_{1}=B$, and $V=\bigcup_{n=1}^{\infty} B_{n}$. Pick $\left(f_{1}, S_{1}, Q\right) \in \mathcal{G}$ with $f_{1}|B=g| B$. This can be done by observing that $\emptyset, Q \in \mathcal{C}$ so, given $(f, S, D) \in \mathcal{F}$, we have $(f \mid S, S, \emptyset) \in \mathcal{F}$ and any map $h:(S \cap \emptyset) \cup B \cup Q \rightarrow K$ extending $g \mid B$ is an extension of $g \mid(S \cap \emptyset)$, which implies the existence of $f_{1}$. Given $\left(g_{n}, S_{n}, A_{n} \cap B_{n}\right) \in \mathcal{G}$ so that $g_{n}\left|A_{n} \cap B_{n}=g\right| A_{n} \cap B_{n}$, define

$$
h:\left(S_{n}-\operatorname{Int}_{Q} A_{n}\right) \cup A_{n} \cap B_{n} \cup A_{n+1} \cap B_{n+1} \rightarrow K
$$

as

$$
h\left|\left(S_{n}-\operatorname{Int}_{Q} A_{n}\right) \cup A_{n} \cap B_{n}=g_{n}\right|\left(S_{n}-\operatorname{Int}_{Q} A_{n}\right) \cup A_{n} \cap B_{n}
$$

and

$$
h\left|A_{n+1} \cap B_{n+1}=g\right| A_{n+1} \cap B_{n+1} .
$$

Since $\left(S_{n}-\operatorname{Int}_{Q} A_{n}\right)=S_{n} \cap\left(Q-\operatorname{Int}_{Q} A_{n}\right)$ and $Q-\operatorname{Int}_{Q} A_{n} \in \mathcal{C}$, there is $\left(g_{n+1}, S_{n+1}\right.$, $\left.A_{n} \cap B_{n} \cup A_{n+1} \cap B_{n+1}\right) \in \mathcal{G}$ so that $S_{n+1} \subset S_{n}$ and

$$
\begin{aligned}
& g_{n+1} \mid\left(S_{n+1}-\operatorname{Int}_{Q} A_{n}\right) \cup A_{n} \cap B_{n} \cup A_{n+1} \cap B_{n+1} \\
& \quad=h \mid\left(S_{n+1}-\operatorname{Int}_{Q} A_{n}\right) \cup A_{n} \cap B_{n} \cup A_{n+1} \cap B_{n+1} .
\end{aligned}
$$

Replace $g_{n+1}$ with $g_{n+1} \mid S_{n+1} \cup A_{n+1} \cap B_{n+1}$.

The direct limit $g^{\prime}$ of $g_{n} \mid X$ extends $g \mid A$. Notice that $g^{\prime} \mid X-A$ is continuous, as for each $x \in X-A$ there is $n>0$ with $x \in X-A_{n} \subset X-\operatorname{Int}_{Q} A_{n}$ and $g^{\prime}\left|X-\operatorname{Int}_{Q} A_{n}=g_{n}\right| X-\operatorname{Int}_{Q} A_{n}$. Also, $g^{\prime}$ is continuous at points of $A$, as for each $a \in A$ there is $n>0$ such that $a \in \operatorname{Int}_{Q}\left(A_{n} \cap B_{n}\right)$ and

$$
g^{\prime}\left|X \cap\left(\operatorname{Int}_{Q}\left(A_{n} \cap B_{n}\right)\right)=g\right| X \cap\left(\operatorname{Int}_{Q}\left(A_{n} \cap B_{n}\right)\right) .
$$

Corollary 3.3. Let $K$ be a $C W$ complex, let $\mathcal{C}$ be $a \cup \cap$-closed basis of $Q$, and let $X$ be a subset of $Q$. Then $K \in A E(X)$ iff any map $g:(X \cap B) \cup D \rightarrow K$, where $B, D \in \mathcal{C}$, extends over $X \cup D$.

Proof. Suppose $K \in A E(X)$ and $g:(X \cap B) \cup D \rightarrow K$ is a map, where $B, D \in \mathcal{C}$. Notice that $(X \cap B) \cup D$ is closed in $X \cup D$. Extend $g$ over a closed neighborhood $N$ of $(X \cap B) \cup D$ in $X \cup D$. Let $G: N \rightarrow K$ be such an extension. Extend $G \mid X \cap N$ to $h: X \rightarrow K$ and define $g^{\prime}: X \cup D \rightarrow K$ as $g^{\prime} \mid X=h$ and $g^{\prime} \mid(X \cap B) \cup D=g$.

Consider the family of all triples $(f, X, D)$ such that $f: X \cup D \rightarrow K$ is a map. This family satisfies the conditions of Theorem 3.2, which implies $K \in A E(X)$.

In order to apply Theorem 3.2 we need the following construction:

Lemma 3.4. Suppose $L$ is a countable subcomplex of a $C W$ complex $K, a: T \rightarrow L$ is a map from a subset $T$ of $Q, X \subset T$, and $\mathcal{C}$ is a $\cup \cap$-closed basis of $Q$. If $K \in$ $A E(X)$, then there is a countable family $\operatorname{Exp}(a, X, L, K)$ of triples $\left(f^{\prime}, S^{\prime}, D\right)$ and a countable subcomplex $K(a)$ of $K$ such that the following conditions are satisfied:

a. $(a, T, \emptyset) \in \operatorname{Exp}(a, X, L, K)$.

b. If $\left(f^{\prime}, S^{\prime}, D\right) \in \operatorname{Exp}(a, X, L, K)$, then $f^{\prime}: S^{\prime} \cup D \rightarrow K(a)$ is a map, $X \subset S^{\prime}$, $D \in \mathcal{C}, f^{\prime}(D) \subset L$, and $S^{\prime}$ is open in $Q$ if $T$ is open in $Q$. 
c. $\left(f \mid\left(S^{\prime} \cap S\right) \cup C, S^{\prime} \cap S, C\right) \in \operatorname{Exp}(a, X, L, K)$ if $(f, S, D) \in \operatorname{Exp}(a, X, L, K)$ and $\left(f^{\prime}, S^{\prime}, D^{\prime}\right) \in \operatorname{Exp}(a, X, L, K)$, and $C \subset D^{\prime} \cap D, C \subset \mathcal{C}$,

d. Given $(f, S, D) \in \operatorname{Exp}(a, X, L, K)$ and given an extension

$$
g:((S \cap B) \cup D \cup C, C) \rightarrow(K(a), L)
$$

of $f \mid(S \cap B) \cup D$, where $B, C \in \mathcal{C}$ with $B \cap C=\emptyset$, there is $\left(f^{\prime}, S^{\prime}, D \cup C\right) \in$ $\operatorname{Exp}(a, X, L, K)$ so that $S^{\prime} \subset S$ and

$$
g\left|\left(\left(S^{\prime} \cap B\right) \cup D \cup C, C\right) \simeq f^{\prime}\right|\left(\left(S^{\prime} \cap B\right) \cup D \cup C, C\right)
$$

$$
\text { in }(K(a), L) \text {. }
$$

Proof. Suppose $B, C, D \in \mathcal{C}$ and $B \cap C=\emptyset$. Suppose $b: S \cup D \rightarrow K$ is a map so that $b(D \cap C) \subset L$ and $X \subset S$. There are countably many extensions $b_{n}: C \rightarrow L$ of $b \mid D \cap C$ so that any extension $h: C \rightarrow L$ of $b \mid D \cap C$ is homotopic rel. $D \cap C$ (in $L$ ) to one of $b_{n}$. Define $f_{n}:(S \cap B) \cup D \cup C \rightarrow K$ as the extension of $b \mid(S \cap B) \cup D$ satisfying $f_{n} \mid C=b_{n}$. Since $B \cap C=\emptyset, f_{n}$ exists. By Lemma 2.5 (used with one term in the join being empty) there are an open neighborhood $S_{n}$ of $X \cup(S \cap B) \cup D \cup C$ in $S \cup D \cup C$ and an extension $F_{n}: S_{n} \rightarrow K$ of $f_{n}$. Replace $S_{n}$ with $S \cap S_{n}$. Let $\alpha(b)$ be the collection of all $\left(F_{n} \mid S_{n} \cup D \cup C, S_{n}, D \cup C\right)$ with all possible choices of $D, C$, and $B$. Clearly, $\alpha(b)$ is countable. Let $K_{b}$ be a countable subcomplex of $K$ containing $L$ and containing $F_{n}\left(S_{n} \cup D \cup C\right)$ for all $n$ and all possible choices of $D, C$ (see 2.1.7).

Next, given a countable family $F$ of triples $(f, S, D), \beta(F)$ is defined as all triples $\left(f \mid\left(S \cap S^{\prime}\right) \cup C, S \cap S^{\prime}, C\right)$ so that $(f, S, D) \in F$ and $\left(f^{\prime}, S^{\prime}, D^{\prime}\right) \in F$, and $C \subset D^{\prime} \cap D$, $C \in \mathcal{C}$.

Define families $\mathcal{F}_{n}$ and subcomplexes $K_{n}, n \geq 1$, by induction as follows:

1. $\mathcal{F}_{1}=\{(a, T, \emptyset)\}, K_{1}=K_{a}$.

2. $\mathcal{F}_{n+1}=\alpha\left(\mathcal{F}_{n}\right) \cup \beta\left(\mathcal{F}_{n}\right), K_{n+1}=\bigcup_{(f, S, D) \in \mathcal{F}_{n}} K_{f}$.

Notice that $\operatorname{Exp}(a, X, L, K)=\bigcup_{n=1}^{\infty} \mathcal{F}_{n}$ and $K(a)=\bigcup_{n=1}^{\infty} K_{n}$ satisfy the desired conditions.

The following theorem is useful when applying results of this paper to arbitrary, not necessarily countable, CW complexes.

Theorem 3.5. Let $K$ be a $C W$ complex, and let $X$ be a separable, metrizable space. If $K \in A E(X)$ and $L$ is a countable subcomplex of $K$, then there is a countable subcomplex $M$ of $K$ which contains $L$ and is an absolute extensor of $X$.

Proof. Assume $X$ is a subset of the Hilbert cube $Q$. Let $\mathcal{C}$ be a countable family of closed subsets of the Hilbert cube $Q$ which is a $\cup \cap$-closed basis (see 3.1). Let $M_{1}=$ $L$ and let $\mathcal{U}_{1}$ consist of a single triple $(c, Q, \emptyset)$, where $c: Q \rightarrow M_{1}$ is a constant map. Suppose $M_{n}$ is a countable subcomplex of $K$, and $\mathcal{U}_{n}$ is a countable set of triples $(f, U, D)$, where $U$ is an open set in $Q$ containing $X, D \in \mathcal{C}$, and $f: U \cup D \rightarrow M_{n}$ is a map. We create (using Lemma 3.4) a new countable family $\mathcal{U}^{\prime}$ as the union of all $\operatorname{Exp}\left(f, X, M_{n}, K\right),(f, U, D) \in \mathcal{U}_{n}$. Now, as in 3.4, we find a countable subcomplex $M_{n+1}$ of $K$ containing $M_{n}$ and containing $K(f)$ for any $(f, U, D) \in \mathcal{U}^{\prime}$. Define $\mathcal{U}_{n+1}$ by $\mathcal{U}_{n+1}=\mathcal{U}^{\prime} \cup \mathcal{U}_{n}$. By Theorem 3.2 (more details below), the intersection $X^{\prime}=\bigcap_{(f, U, D) \in \mathcal{U}} U$, where $\mathcal{U}=\bigcup_{n=1}^{\infty} \mathcal{U}_{n}$, satisfies $M \in A E\left(X^{\prime}\right)$ if $M=\bigcup_{n=1}^{\infty} M_{n}$. 
Therefore, $M \in A E(X)$. Indeed, suppose $(f, U, D) \in \mathcal{U}, f: U \cup D \rightarrow M_{n}$ for some $n$, and $g:(U \cap B) \cup D \cup C \rightarrow M$ is an extension of $f \mid(U \cap B) \cup D$, where $D, C \in \mathcal{C}$ and $B \cap C=\emptyset$. Then $g(C)$ is a compact subset of $M$; therefore $g(C) \subset M_{m}$ for some $m>n$. Since $\mathcal{U}_{m+1}$ contains a subset of the form $\operatorname{Exp}\left(f, X, M_{m}, K\right)$, there is $\left(f^{\prime}, U^{\prime}, D \cup C\right) \in \mathcal{U}_{m+1}$ so that $f^{\prime} \mid(S \cap B) \cup D \cup C$ is homotopic to $g$ in $M_{m+1}$.

Theorem 3.6 (Completion Theorem of W.Olszewski $\left.\mathrm{Ol}_{1}\right)$. Let $K$ be a countable $C W$ complex. If $X$ is a separable metrizable space, and $K$ is an absolute extensor of $X$, then there is a completion $X^{\prime}$ of $X$ such that $K \in A E\left(X^{\prime}\right)$.

Proof. Assume $X$ is a subset of the Hilbert cube $Q$. Let $\mathcal{C}$ be a countable family of closed subsets of the Hilbert cube $Q$ which is a $\cup \cap$-closed basis (see 3.1). Let $\mathcal{U}_{1}$ consist of a single triple $(c, Q, \emptyset)$, where $c: Q \rightarrow K$ is a constant map. Suppose $\mathcal{U}_{n}$ is a countable set of triples $(f, U, D)$, where $U$ is an open set in $Q$ containing $X$, $D \in \mathcal{C}$, and $f: U \cup D \rightarrow K$ is a map. We create (using Lemma 3.4) a new countable family $\mathcal{U}_{n+1}$ as the union of all $\operatorname{Exp}(f, X, K, K),(f, U, D) \in \mathcal{U}_{n}$. By Theorem 3.2 the intersection $X^{\prime}=\bigcap_{(f, U, D) \in \mathcal{U}} U$, where $\mathcal{U}=\bigcup_{n=1}^{\infty} \mathcal{U}_{n}$, satisfies $K \in A E\left(X^{\prime}\right)$.

Theorem 3.7 (Generalized Eilenberg-Borsuk Theorem). Let $L$ be a countable $C W$ complex. If $X$ is a separable metrizable space and $K * L$ is an absolute extensor of $X$ for some $C W$ complex $K$, then for any map $r: A \rightarrow K, A$ closed in $X$, there is an extension $r^{\prime}: U \rightarrow K$ of $f$ over an open set $U$ such that $L \in A E(X-U)$.

Proof. Let $\pi_{K}: K * L-L \rightarrow K, \pi_{L}: K * L-K \rightarrow L$, and $\pi: K * L \rightarrow[0,1]$ be projections.

Since, by Part 2 of Lemma 2.1, being an absolute extensor is a property of the homotopy type, we may assume that both $K$ and $L$ are metric simplicial complexes. Let $\mathcal{C}=\left\{C_{i}\right\}_{i \geq 1}$ be a countable family of closed subsets of the Hilbert cube $Q$ which is a $\cup \cap$-closed basis. Suppose $A$ is a closed subset of $X$, and $r: A \rightarrow K$ is a map. Extend $r$ over a closed neighborhood $N_{1}$ of $A$ in $X$ and let $f_{1}: N_{1} \rightarrow K$ be an extension of $r$. Put $M_{1}=X-\operatorname{Int}_{X} N_{1}$, and let $g_{1}: M_{1} \rightarrow L$ be a constant map.

Suppose we have a finite sequence of maps $f_{i}: N_{i} \rightarrow K, 1 \leq i \leq n$, such that the following conditions are satisfied if $M_{i}=X-\operatorname{Int}_{X} N_{i}$ :

1. $N_{i+1}$ is a closed neighborhood of $N_{i}$ in $X$.

2. $f_{i+1} \mid N_{i} \simeq f_{i}$.

3. There is a map $g_{i}: M_{i} \cup D_{i} \rightarrow L, D_{i} \in \mathcal{C}$.

4. There are maps $g_{i, j, k, m, r}:\left(M_{i} \cap C_{j}\right) \cup C_{k} \cup C_{m} \rightarrow L, j, k, m \geq 1$, such that $C_{j} \cap C_{m}=\emptyset, C_{k} \subset D_{i}, g_{i, j, k, m, r}\left|\left(M_{i} \cap C_{j}\right) \cup C_{k} \simeq g_{i}\right|\left(M_{i} \cap C_{j}\right) \cup C_{k}$, and for any extension $g:\left(M_{i} \cap C_{j}\right) \cup C_{k} \cup C_{m} \rightarrow L$ of $g_{i} \mid\left(M_{i} \cap C_{j}\right) \cup C_{k}$, there is $(i, j, k, m, r)$ such that $g_{i, j, k, m, r} \simeq g$.

Consider the set of all 5 -tuples $(i, j, k, r, m), i \leq n$, so that there is no $p>k$ with $C_{k} \cup C_{m} \subset D_{p}$ and

$$
g_{p}\left|\left(M_{p} \cap C_{j}\right) \cup C_{k} \cup C_{m} \simeq g_{i, j, k, m, r}\right|\left(M_{p} \cap C_{j}\right) \cup C_{k} \cup C_{m} .
$$

Among all those 5-tuples choose one minimizing the sum $i+j+k+r+m$. Put $g_{i, j, k, m, r}=g, B=C_{j}, C=C_{m}$. Notice that $\left(M_{n} \cap B\right) \cup C_{k} \cup C$ is closed in $Y=X \cup C_{k} \cup C$. By Lemma 2.5 there is a map $h: Y \rightarrow K * L$ such that $\pi_{K} \circ h \mid N_{n} \simeq f_{n}$ and $\pi_{L} \circ h\left|\left(M_{n} \cap B\right) \cup C_{k} \cup C=g\right|\left(M_{n} \cap B\right) \cup C_{k} \cup C$. Let $N_{n+1}$ be a closed neighborhood of $N_{n} \cup\left(X \cap h^{-1}(K)\right)$ in $X, N_{n+1} \subset X \cap h^{-1}(K * L-L)$, and 
let $M_{n+1}=X-\operatorname{Int}_{X}\left(N_{n+1}\right)$. Put $D_{n+1}=C_{k} \cup C, g_{n+1}=\pi_{L} \circ h \mid M_{n+1} \cup C \cup C_{k}$ : $M_{n+1} \cup C \cup C_{k} \rightarrow L$ and $f_{n+1}=\pi_{K} \circ h \mid N_{n+1}$.

Using the above construction we create a countable family of triples $\left\{\left(g_{i}, M_{i}, D_{i}\right)\right\}$ so that $\left\{\left(g_{i} \mid M_{i} \cup D_{i} \cap C_{j}, M_{i}, D_{i} \cap C_{j}\right)\right\}$ satisfies the hypotheses of Theorem 3.2. Thus, $Y=\bigcap_{i=1}^{\infty} M_{i}$ satisfies $L \in A E(Y)$. Put $U=X-Y$ and notice that $U=\bigcup_{i=1}^{\infty} N_{i}$, which implies that $f$ extends over $U$.

Theorem 3.8. Let $K, L$ be countable $C W$ complexes. If $X$ is a separable metrizable space, and $K * L$ is an absolute extensor of $X$, then there is a subset $Y$ of $X$ such that $K \in A E(Y)$ and $L \in A E(X-Y)$.

Proof. Let $\mathcal{C}$ be a countable family of closed subsets of the Hilbert cube $Q$ which is a $\cup \cap$-closed basis. Let $\mathcal{U}_{1}$ consist of a single triple $(f, X, Q)$, where $f$ is a constant map from $Q$ to $K$. Given a countable family of triples $\mathcal{U}=\{(f, U, D)\}$ such that $U$ is open in $X, D \in \mathcal{C}, f: U \cup D \rightarrow K$ is a map, and $L \in A E(X-U)$, we create a new countable family $\mathcal{U}^{\prime}=\left\{\left(f^{\prime}, U^{\prime}, D^{\prime}\right)\right\}$ of triples with the property that, given any $B, C, D \in \mathcal{C}, B \cap C=\emptyset$ and given any $g:(U \cap B) \cup D \cup C \rightarrow K$ such that $g|(U \cap B) \cup D=f|(U \cap B) \cup D$, then there is $\left(f^{\prime}, U^{\prime}, D \cup C\right) \in \mathcal{U}^{\prime}$ so that $U^{\prime} \subset U$, $f^{\prime}\left|\left(U^{\prime} \cap B\right) \cup D \simeq g\right|\left(U^{\prime} \cap B\right) \cup D$, and $L \in A E\left(X-U^{\prime}\right)$. This is done as in Lemma 3.4 with the help of the following observation: if $g:(U \cap B) \cup D \cup C \rightarrow K$ is a map, then we extend it first to $G: N \rightarrow K$ for some closed neighborhood $N$ of $(U \cap B) \cup D \cup C$ in $U \cup D \cup C$. Since $N \cap U$ is closed in $U$ and $K * L \in A E(U)$, Theorem 3.7 guarantees the existence of an open neighborhood $U^{\prime}$ of $N \cap U$ in $U$ such that $G \mid N \cap U$ extends to $h: U^{\prime} \rightarrow K$ and $L \in A E\left(U-U^{\prime}\right)$. The map $f^{\prime}: U^{\prime} \cup D \cup C \rightarrow K$, $f^{\prime}\left|U^{\prime}=h, f^{\prime}\right|(U \cap B) \cup D \cup C=g$, can be used as in 3.4 to add a new element to $\mathcal{U}^{\prime}$. Notice that $L \in A E\left(X-U^{\prime}\right)$. Indeed, $U-U^{\prime}$ can be expressed as a countable union of closed sets in $X$. Since $X-U^{\prime}=(X-U) \cup\left(U-U^{\prime}\right)$, we are done.

Define $\mathcal{U}_{n}$ for $n>1$ by $\mathcal{U}_{n}=\mathcal{U}_{n-1}^{\prime}$. The intersection $Y$ of the domains of all maps in $\bigcup_{n=1}^{\infty} \mathcal{U}_{n}$ satisfies $K \in A E(Y)$ by Theorem 3.2. Notice that $L \in A E(X-Y)$.

The following result is related to Theorem 2.7:

Theorem 3.9. Let $K, L$ be countable $C W$ complexes and let $X$ be a separable metrizable space. If for any map $f: A \rightarrow K, A$ closed in an open subset $U$ of $X$, there is an extension $f^{\prime}: U^{\prime} \rightarrow K$ so that $L \in A E\left(U-U^{\prime}\right)$, then $K * L$ is an absolute extensor of $X$.

Proof. As in the proof of 3.8, we can find $Y \subset X$ so that $K \in A E(Y)$ and $L \in$ $A E(X-Y)$. By Theorem 1.2 of $\left[\mathrm{Dy}_{2}\right.$ ] (see Conjecture 1.17), $K * L \in A E(X)$.

Theorem 3.10. Let $X$ be a separable metrizable space, and let $K_{i}, i \leq n$, be countable $C W$ complexes. If, for all $i \leq n, \Sigma^{2} K_{i} \in A E(X), \Sigma K_{i} \notin A E(X)$, then there are a closed subset $Y$ of $X$ such that, for all $i \leq n, \Sigma K_{i} \in A E(Y), K_{i} \notin$ $A E(Y)$.

Proof. Fix $j \leq n$. Since $S^{0} * K_{j}=\Sigma K_{j} \notin A E(X)$, there is a closed subset $A$ of $U$, $U$ open in $X$, and a map $f: A \rightarrow S^{0}$ such that, for any extension $F: U^{\prime} \rightarrow S^{0}$ of $f$ over an open neighborhood $U^{\prime}$ of $A$ in $U, K_{j} \notin A E\left(U-U^{\prime}\right)$ (see Theorem 3.9). On the other hand, $S^{0} *\left(\bigvee_{i=1}^{n} \Sigma K_{i}\right)$ is homotopy equivalent to $\bigvee_{i=1}^{n} \Sigma^{2} K_{i} \in A E(U)$, 
which implies, by Theorem 3.7, that there is an extension $F: U^{\prime} \rightarrow S^{0}$ of $f$ over an open neighborhood $U^{\prime}$ of $A$ in $U$ such that $\bigvee_{i=1}^{n} \Sigma K_{i} \in A E\left(U-U^{\prime}\right)$. Express $U-U^{\prime}$ as the union of countably many closed sets $Y_{U, i}$ in $X$. There is $k$ such that $K_{j} \notin A E\left(Y_{U, k}\right)$. Put $Y_{j}=Y_{U, i}$. Finally, put $Y=\bigcup_{i=1}^{n} Y_{i}$.

Theorem 3.11. Let $X$ be a separable metrizable space, and let $K * L$ be an absolute extensor of $X$ for some metric simplicial complexes $K$ and $L$. If $K$ and $L$ are countable, then for any map $f: A \rightarrow K * L, A$ closed in $X$, there is an extension $g: X \rightarrow K * L$ of $f$ such that $K \in A E\left(g^{-1}(K)-A\right)$ and $L \in A E\left(g^{-1}(L)-A\right)$.

Proof. Let $\pi: K * L \rightarrow K *\{v\}$ be the natural projection. Extend $\pi \circ f: A \rightarrow K *\{v\}$ to $F: X \rightarrow K *\{v\}$ so that $L \in A E\left(F^{-1}(v)-A\right.$ ) (see Lemma 2.5). Extend $\pi_{L} \circ f: f^{-1}(L) \rightarrow L$ to $G: F^{-1}(v) \rightarrow L$. Now, extend $G \cup f \mid\left(A-f^{-1}(K)\right)$ to $H: U \rightarrow L$ for some open neighborhood $U$ of $F^{-1}(v) \cup\left(A-f^{-1}(K)\right)$ in $X-f^{-1}(K)$.

Let $p r: K * L \rightarrow\{v\} * L$ be the natural projection. Extend $p r \circ f: A \rightarrow\{v\} * L$ to $J: X \rightarrow\{v\} * L$ so that $K \in A E\left(J^{-1}(v)-A\right.$ ) (see Lemma 2.5). Extend $p r_{K} \circ f: f^{-1}(K) \rightarrow K$ to $P: J^{-1}(v) \rightarrow K$. Now, extend $P \cup f \mid\left(A-f^{-1}(L)\right)$ to $R: V \rightarrow K$ for some open neighborhood $V$ of $J^{-1}(v) \cup\left(A-f^{-1}(L)\right)$ in $X-f^{-1}(L)$.

Let $\pi: K * L \rightarrow[0,1]$ be the projection. Extend the composition $\pi \circ f$ over $(X-U) \cup(X-V) \cup A$ by sending $X-U$ to 0 , and by sending $X-V$ to 1 . Extend the resulting map to $\alpha: X \rightarrow[0,1]$ so that $\alpha^{-1}(1)=X-V$ and $\alpha^{-1}(0)=X-U$ (see Lemma 2.0). Now, use Lemma 2.4.

Corollary 3.12. Let $X$ be a separable metrizable space, and let $K$ be a countable metric simplicial complex. For any map $f: A \rightarrow C o n e(K), A$ closed in $X$, there is an extension $g: X \rightarrow C o n e(K)$ of $f$ such that $K \in A E\left(g^{-1}(K)-A\right)$.

\section{Applications to COHOMOlogical Dimension theory}

Theorem 4.1. Suppose $G_{i}, \ldots, G_{n}$ are countable, non-trivial, abelian groups, and $k>0$. For any separable metrizable space $X$ of finite dimension $\operatorname{dim} X>0$, there is a closed subset $Y$ of $X$ with

$$
\operatorname{dim}_{G_{i}} Y=\max \left(\operatorname{dim}_{G_{i}} X-k, 1\right)
$$

for $i=1, \ldots, n$.

Proof. It suffices to consider the case $k=1$. Choose a closed subset $A$ of $X$ of dimension 1 (apply Theorem 3.10 or use Theorem 1.27). Let $k_{i}=\operatorname{dim}_{G_{i}} X$. Create a new list $H_{1}, \ldots, H_{m}$ consisting of groups $G_{i}$ so that $k_{i}>2$. Let $M_{i}(q)$ be the Moore space $M\left(H_{i}, q\right)$. For $i \leq m$, let $K_{i}=M\left(H_{i}, k_{i}-2\right)$. By Theorem 3.10, there is a closed subset $B$ of $X$ such that $K_{i} \notin A E(B)$, but $\Sigma\left(K_{i}\right) \in A E(B)$ for all $i \leq m$. By Theorem 1.28, $\operatorname{dim}_{H_{i}} B=k_{i}-1$ for all $i \leq m$. Put $Y=A \cup B$.

Corollary 4.2. Suppose $G_{i}, i \geq 1$, are countable, non-trivial, abelian groups, and $k>0$. For any separable metrizable space $X$ of finite dimension $\operatorname{dim} X>0$ there is a $\sigma$-closed subset $Y$ of $X$ such that

$$
\operatorname{dim}_{G_{i}} Y=\max \left(\operatorname{dim}_{G_{i}} X-k, 1\right)
$$

for $i \geq 1$. 
Proof. Let $F_{i}=\bigoplus_{j=i}^{\infty} G_{j}$. By Theorem 4.1 there is a closed subset $Y_{n}$ of $X$ such that $\operatorname{dim}_{G} Y_{n}=\max \left(\operatorname{dim}_{G} X-k, 1\right)$ for all $G \in\left\{G_{1}, \ldots, G_{n}, F_{n}\right\}$. Let $Y$ be the union of all $Y_{n}, n \geq 1$.

Theorem 4.3. Suppose $G_{i}, \ldots, G_{n}$ are non-trivial, abelian groups and $k>0$. For any $\sigma$-compact metrizable space $X$ of finite dimension $\operatorname{dim} X>0$, there is a compact subset $Y$ of $X$ with

$$
\operatorname{dim}_{G_{i}} Y=\max \left(\operatorname{dim}_{G_{i}} X-k, 1\right)
$$

for $i=1, \ldots, n$.

Proof. By the Bockstein Theorem $[\mathrm{Ku}]$, for each abelian group $G$ there is a countable abelian group $H$ such that $\operatorname{dim}_{G} X=\operatorname{dim}_{H} X$ for all compacta $X$. Now, use Theorem 4.1.

Corollary 4.4. For any $\sigma$-compact metrizable space $X$ of finite dimension $\operatorname{dim} X>$ 0 , and for any $k>0$, there is a $\sigma$-compact subset $Y$ of $X$ with

$$
\operatorname{dim}_{G} Y=\max \left(\operatorname{dim}_{G} X-k, 1\right)
$$

for any abelian group $G$.

Proof. It suffices to consider compact $X$ only. By the Bockstein Theorem $\mathrm{Ku}$ ] there is a sequence $\mathcal{B G}=\left\{B_{1}, \ldots, B_{n}, \ldots\right\}$ of countable abelian groups such that for each abelian group $G$ there is a subset $\sigma(G)$ of $\mathcal{B G}$ with the property that $\operatorname{dim}_{G} X=\sup \left\{\operatorname{dim}_{H} X \mid H \in \sigma(G)\right\}$ for all compacta $X$. Now, use Corollary 4.2 .

Theorem 4.5. Suppose $G$ is a countable ring with unity, $k$ is an integer, and $X$ is a separable metrizable space of finite dimension. Then, $\operatorname{dim}_{G} X \leq n$, where $n \geq 2$, if and only if any map $f: A \rightarrow K(G, k), A$ closed in an open subset $U$ of $X$, extends over a neighborhood $U^{\prime}$ of $A$ in $U$ with

$$
\operatorname{dim}_{G}\left(U-U^{\prime}\right) \leq n-k-1
$$

Proof. Suppose any map $f: A \rightarrow K(G, k), A$ closed in an open subset $U$ of $X$, extends over a neighborhood $U^{\prime}$ of $A$ in $U$ with $\operatorname{dim}_{G}\left(U-U^{\prime}\right) \leq n-k-1$. By Theorem 3.9, $K(G, k) * K(G, n-k-1) \in A E(X)$, and by Theorem 3.8 there is a subset $Y$ of $X$ with $\operatorname{dim}_{G} Y \leq k$ and $\operatorname{dim}_{G}(X-Y) \leq n-k-1$, which implies $\operatorname{dim}_{G} X \leq n$ in view of Theorem 1.4 of $\left|\mathrm{Dy}_{2}\right|$ ).

Suppose $\operatorname{dim}_{G} X \leq n$, and $f: A \rightarrow K(G, k), A$ closed in an open subset $U$ of $X$. Notice that the homotopy groups of $K(G, k) * K(G, n-k-1)$ are trivial in the range from 0 to $n-1$, and are $G$-modules starting from $n$. Therefore,

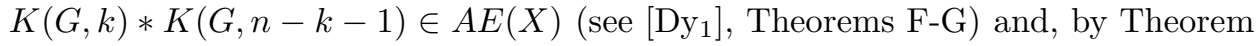
3.7, $f$ extends over a neighborhood $U^{\prime}$ of $A$ in $U$ with $\operatorname{dim}_{G}\left(U-U^{\prime}\right) \leq n-k-1$.

Theorem 4.6. Suppose $G$ is an abelian group, and $X$ is a separable metrizable space with $\operatorname{dim}_{G} X<\infty$. Then, for any countable subgroup $G^{\prime}$ of $G$, there is a countable subgroup $H$ of $G$ containing $G^{\prime}$ such that $\operatorname{dim}_{G} X=\operatorname{dim}_{H} X$.

Proof. Let $\operatorname{dim}_{G} X=n$. Arrange all countable subgroups of $G$ in a direct system $\left\{G_{s} \mid h_{s, s^{\prime}}, S\right\}$ so that $s<s^{\prime}$ means that $G_{s} \subset G_{s^{\prime}}$, and $h_{s, s^{\prime}}: G_{s} \rightarrow G_{s^{\prime}}$ is the inclusion. Let $N$ be the simplicial complex whose set of vertices is $S$ and $\left\langle s_{0}, \ldots, s_{m}\right\rangle$ is an $m$-simplex of $N$ iff $\left.s_{0}>\ldots\right\rangle s_{m}$. As in Lemma 2.6 of Dy , 
$N$ is contractible and there are a $\mathrm{CW}$ complex $L$ of the type $K(G, n)$ and a map $\pi: L \rightarrow N$ such that for each simplex $\Delta$ of $N, \pi^{-1}(\Delta)$ is a countable subcomplex of $L$ which is of type $K\left(G_{s}, n\right)$, where $s$ is the minimal vertex of $\Delta$. Moreover, the inclusion $\pi^{-1}\left(\Delta^{\prime}\right) \rightarrow \pi^{-1}(\Delta)$ induces $h_{s, s^{\prime}}: G_{s} \rightarrow G_{s^{\prime}}$ on the $n$-th homotopy groups. Given a countable subcomplex $L^{\prime}$ of $L$, one can find a vertex $v$ of $N$ and a countable subcomplex $M$ of $N$ so that $w>v$ for all vertices $w$ of $M$, and $\pi\left(L^{\prime}\right) \subset M$. Now, $M * v$ is a subcomplex of $N, \pi^{-1}(M * v) \supset L^{\prime}$, and $\pi^{-1}(M * v)$ is of type $K\left(G_{v}, n\right)$. Choose $v(1)$ so that $G_{v(1)}=G^{\prime}$, and let $N_{1}=\{v(1)\}$. Using induction, we can construct an increasing sequence $N_{i}$ of countable subcomplexes of $N$, and an increasing sequence $L_{i}$ of countable subcomplexes of $L$ such that the following conditions are satisfied:

1. $\pi^{-1}\left(N_{i}\right) \subset L_{i}$.

2. $L_{i} \in A E(X)$.

3. $\pi\left(L_{i}\right) \subset N_{i+1}$.

4. $N_{i} * v_{i} \subset N_{i+1}$ for some vertex $v_{i}$ of $N_{i+1}$.

Let $M=\bigcup_{i=1}^{\infty} N_{i}$. Notice that $\pi^{-1}(M)=\bigcup_{i=1}^{\infty} L_{i} \in A E(X)$ is of type $K(H, n)$ for some countable subgroup $H$ of $G$ which contains $G^{\prime}$.

In the following two results $H_{k}(X ; G)$ stands for the $k$-th reduced Steenrod homology group of $X$ if $X$ is compact, and stands for the $k$-th reduced SteenrodSitnikov homology group of $X$ if $X$ is not compact. (See [Mas] for the properties of Steenrod homology groups.)

The purpose of Theorem 4.7 is to expose difficulties one has to face when generalizing results of the cohomological dimension theory of compacta to separable, metrizable spaces. It was shown by Dranishnikov, Repovš, and Ščepin $\left[\mathrm{D}-\mathrm{R}-\mathrm{S}_{2}\right.$. that the Bockstein Theorem does not hold for separable, metrizable spaces. Our result is even stronger.

Theorem 4.7. Suppose $G$ is a countable abelian group, and $A_{p}$ is the ring of $p$ adic integers for some prime number $p$. There is a separable space $W$ of dimension 2 such that

$$
\operatorname{dim}_{G} W \neq \operatorname{dim}_{A_{p}} W \text {. }
$$

Proof. If $G$ is a torsion group or $G$ / Tor $G$ is divisible by $p$, then there is a compactum $W$ of dimension 2 such that $\operatorname{dim}_{G} W=1$ and $\operatorname{dim}_{A_{p}} W=2$ (see [Dra $]$ ). Assume $G / \operatorname{Tor} G$ is not divisible by $p$. Express $(G / \operatorname{Tor} G) \otimes \mathbf{Z}_{(p)}$ as $H \oplus D$, where $D$ is a maximal divisible subgroup (a direct sum of copies of rationals) of $(G / \operatorname{Tor} G) \otimes \mathbf{Z}_{(p)}$. Notice that $H \neq 0$, and $\bigcap_{n=1}^{\infty} p^{k} H=\{0\}$. By Lemma 4.8 there is a two-point subset $D$ of the $p$-adic solenoid $C$ such that $H_{1}(C / D ; H)=0$. Let $X$ be a $(C, D)$-continuum of dimension 1, i.e. any map $f: A \rightarrow D, A$ closed in $X$, extends to $f^{\prime}: X \rightarrow C$ (see D-R-S $_{2}$ ). As in D-R-S (Propositions 1.3-1.5) one gets that, for every pair of closed subsets $B \subset A$ of $X$, the group $H_{1}(A / B ; H)$ is trivial.

Let $T$ be the subset of $R^{3}$ obtained by connecting every pair $a, b$ of different points in $Q^{3}$ ( $Q$ being the rationals) by a copy $X_{a b}$ of $X$ so that the diameter of $X_{a b}$ is $|a-b|$. We request that $X_{a b} \cap X_{c d}=\{a, b\} \cap\{c, d\}$ if $\{a, b\} \neq\{c, d\}$. Notice that $H_{0}\left(X ; A_{p}\right)=\operatorname{Ext}\left(H^{1}(X ; \mathbf{Z}), A_{p}\right)=0$. Therefore, by Proposition 2.6 of D-R-S,$H_{0}\left(U \cap T ; A_{p}\right)=0$ for every open ball $U$ of $R^{3}$, and, by the Sitnikov 
Duality, $H^{2}\left(U \cap\left(R^{3}-T\right) /\left(\partial U \cap\left(R^{3}-T\right)\right) ; A_{p}\right)=0$. Thus, by Lemma 3.5 of D-R-S $2, \operatorname{dim}_{A_{p}} W=1$. To show that $\operatorname{dim}_{H} W=2$ it suffices to prove $H_{0}(T ; H) \neq$ 0 . Suppose $H_{0}(T ; H)=0$, and choose $D=\{a, b\}, a \neq b \in Q^{3}$. There is a compact subset $Y$ of $T$ such that $H_{0}(D ; H) \rightarrow H_{0}(Y ; H)$ is not a monomorphism. There is an open subset $V$ of $Y-D$ such that $V \subset X_{c d}$ for some $c, d \in Q^{3}$ (use the Baire Category Theorem). Since $H_{1}(Y /(Y-V) ; H)=0$, it follows that $H_{0}(Y-V ; H) \rightarrow H_{0}(Y ; H)$ is a monomorphism, and $H_{0}(D ; H) \rightarrow H_{0}(Y-V ; H)$ is not a monomorphism. As in D-R-S $_{2}$ (Lemma 3.3), one proves that there must be a minimal $Y$ so that $H_{0}(D ; H) \rightarrow H_{0}(Y ; H)$ is not a monomorphism, and that $Y$ must be $D$, a contradiction. By Corollary 2.8 of $\overline{\mathrm{Dy}_{1}}$, and Theorem B of $\overline{\mathrm{Dy}_{1}}$, $2=\operatorname{dim} W \geq \operatorname{dim}_{G} W \geq \operatorname{dim}_{H} W=2$, which completes the proof.

Lemma 4.8. Suppose $G$ is a countable, torsion-free abelian group such that

$$
\bigcap_{n=1}^{\infty} p^{k} G=\{0\}
$$

for some prime number $p$. If $\Sigma_{p}$ is the p-adic solenoid, then there is a two-point subset $D$ of $\Sigma_{p}$ such that $H_{1}\left(\Sigma_{p} / D ; G\right)=0$.

Proof. This lemma is a generalization of Proposition 1.1 of $\left[\mathrm{D}-\mathrm{R}-\mathrm{S}_{2}\right]$, which deals with the case $G=\mathbf{Z}$, and characterizes two-point subsets $D$ of $\Sigma_{p}$ with $H_{1}\left(\Sigma_{p} / D ; \mathbf{Z}\right)$ $=0$ as those homotopic to a pair $a, b$ with $b-a \in A_{p}-\mathbf{Z}_{(p)}$. Our plan is to use ideas of that proposition with the distinction that we have no characterization of two-point subsets $D$ of $\Sigma_{p}$ with $H_{1}\left(\Sigma_{p} / D ; G\right)=0$.

We will find $D=\{0, c\} \subset A_{p}$, where $c=n_{0}+n_{1} p+\ldots+n_{k} p^{k}+\ldots$ and $0 \leq n_{i}<p$ for all $i$ (see [Kob]). Notice that $H_{1}\left(\Sigma_{p} / D ; G\right)=H_{1}\left(\Sigma_{p} \cup I ; G\right)=$ $\check{H}_{1}\left(\Sigma_{p} \cup I ; G\right)$, where $I$ is an interval joining 0 and $c . \Sigma_{p} \cup I$ is the inverse limit of $S^{1} \cup I \leftarrow S^{1} \cup I \leftarrow \ldots$ with each bonding map being a homeomorphism on $I$ and sending $z$ to $z^{p}$ on $S^{1}$. As in $\left[\mathrm{D}-\mathrm{R}-\mathrm{S}_{2}\right]$ (where $G=\mathbf{Z}$ ), $\breve{H}_{1}\left(\Sigma_{p} \cup I ; G\right)$ is the inverse limit of $G \oplus G \leftarrow G \oplus G \leftarrow \ldots$ and the $i$-th homomorphism $\phi_{i}^{i+1}: G \oplus G \rightarrow G \oplus G$ is given by $\phi_{i}^{i+1}(g, h)=\left(p g+n_{i} h, h\right)$. Hence, $\phi_{0}^{k+1}(g, h)=\left(p^{k+1} g+c_{k} h, h\right)$, where $c_{k}=n_{0}+n_{1} p+\ldots+n_{k} p^{k}$. Thus, $\operatorname{Im} \phi_{0}^{k+1}$ can be characterized as pairs $(u, v)$ with $p^{k+1} \mid u-c_{k} v$, and $\operatorname{Im} \phi_{0}^{\infty}=\operatorname{Im}\left(\check{H}_{1}\left(\Sigma_{p} \cup I ; G\right) \rightarrow G \oplus G\right)$ consists of pairs $(u, v)$ with $p^{k+1} \mid u-c_{k} v$ for all $k$.

Let us fix a pair $(u, v) \in G \oplus G-(0,0)$, and let us show that there is at most one $c=n_{0}+n_{1} p+\ldots+n_{k} p^{k}+\ldots \in A_{p}$ with $p^{k+1} \mid u-c_{k} v$ for all $k$. Indeed, suppose $d=m_{0}+m_{1} p+\ldots+m_{k} p^{k}+\ldots \in A_{p}$ and $p^{k} \mid u-d_{k} v$ for all $k$. Choose the smallest $i$ with $n_{i} \neq m_{i}$, and notice that $p^{j+1} \mid\left(u-c_{j} v\right)-\left(u-d_{j} v\right)=\left(d_{j}-c_{j}\right) v$ for all $j \geq i$. Since $d_{j}-c_{j}$ is not divisible by $p^{i+1}$, it follows that $p^{j+1-i} \mid v$. Thus, $v=0$, and, since $p^{k+1} \mid u-c_{k} v$ for all $k$, it follows that $u=0$. Since $G$ is countable, there is a countable subset $S_{0} \subset \Sigma_{p}$ such that if $c \in \Sigma_{p}-S_{0}$ then

$$
\operatorname{Im} \phi_{0}^{\infty}=\operatorname{Im}\left(\check{H}_{1}\left(\Sigma_{p} \cup I ; G\right) \rightarrow G \oplus G\right)
$$

is trivial. Similarly, there is a countable subset $S_{k} \subset \Sigma_{p}$ such that if $c \in \Sigma_{p}-S_{k}$ then

$$
\operatorname{Im} \phi_{k}^{\infty}=\operatorname{Im}\left(\check{H}_{1}\left(\Sigma_{p} \cup I ; G\right) \rightarrow G \oplus G\right)
$$

is trivial. Finally, choose $c \in A_{p}-\bigcup_{i=0}^{\infty} S_{i}$. 


\section{COHOMOlOGiCAl Dimension OF CARTESIAN PRODUCTS}

In 1991, E.Ščepin (private communication) speculated on the connection between $\operatorname{dim}(A * B)$ and $\operatorname{dim}(A \cup B)$. The idea was that there ought to be a similarity between the two expressions. In particular, he conjectured that

$$
1+\operatorname{dim}(A \times B)=\operatorname{dim}(A * B) \geq \operatorname{dim}(A \cup B) .
$$

This conjecture was partially confirmed by A.Dranishnikov Dra7. Namely, he proved that if $X=A \cup B$ is a compactum, then $1+\operatorname{dim}(A \times B) \geq \operatorname{dim}(A \cup B)$ if $\operatorname{dim}(X \times X)=2 \operatorname{dim} X$, and $2+\operatorname{dim}(A \times B) \geq \operatorname{dim}(A \cup B)$ if $\operatorname{dim}(X \times X) \neq$ $2 \operatorname{dim} X$. In 1992, J.Dydak $\mathrm{Dy}_{2}$ proved the following result, which was conceived as a Urysohn-Menger Theorem in cohomological dimension theory:

Theorem 5.1. Suppose $A$ and $B$ are subsets of a metrizable space, and $G$ and $H$ are abelian groups. Then

$$
\operatorname{dim}_{G \otimes H}(A \cup B) \leq \operatorname{dim}_{G} A+\operatorname{dim}_{H} B+1
$$

and

$$
\operatorname{dim}_{G * H}(A \cup B) \leq \operatorname{dim}_{G} A+\operatorname{dim}_{H} B+2 .
$$

Theorem 5.1 was deduced from the analog of the Urysohn-Menger Theorem in extension theory: $K \in A E(A), L \in A E(B)$ implies $K * L \in A E(A \cup B)$. In this paper, we reinterpret Ščepin's idea to mean that if there is an estimate of $\operatorname{dim}_{G}(A \cup B)$ using $\operatorname{dim}_{G} A$ and $\operatorname{dim}_{G} B$, then there ought to be a similar estimate of $\operatorname{dim}_{G}(A * B)=1+\operatorname{dim}_{G}(A \times B)$. Here is an analog of the Menger Theorem in cohomological dimension theory which we obtained using this reasoning:

Theorem 5.2. Suppose $X$ is a metrizable space, $Y$ is a $\sigma$-compact metrizable space, and $G, H$ are abelian groups. Then

$$
\operatorname{dim}_{G \otimes H}(X \times Y) \leq \operatorname{dim}_{G} X+\operatorname{dim}_{H} Y
$$

and

$$
\operatorname{dim}_{G * H}(X \times Y) \leq \operatorname{dim}_{G} X+\operatorname{dim}_{H} Y+1 .
$$

Proof. Suppose $G, H \neq 0$ are abelian groups and $\operatorname{dim}_{G} X=m, \operatorname{dim}_{H} Y=n$.

We may assume $Y$ is compact. First, let us prove that

$$
\operatorname{dim}_{G * H}(X \times Y) \leq m+n+1
$$

Since $G * H=(\operatorname{Tor} G) *($ Tor $H)$ and $\operatorname{dim}_{F} Z \geq \operatorname{dim}_{\text {Tor } F} Z$ for any metrizable space $Z$ (see $\mathrm{Dy}_{1}$, Theorem B), we may assume $\bar{G}=$ Tor $G, H=$ Tor $H$. Since each torsion group is the direct limit of its $\mathbf{p}$-groups, and the torsion product commutes with the direct product, we may assume that $G$ is a p-group and $H$ is a q-group for some primes $\mathbf{p}, \mathbf{q}$. If $\mathbf{p} \neq \mathbf{q}$, then $G * H=0$, and we are done. So, assume $\mathbf{p}=\mathbf{q}$. If $G * H$ is not divisible by $\mathbf{p}$, then either $G$ or $H$ is not divisible by $\mathbf{p}$. In such a case $\operatorname{dim}_{G * H}(X \times Y)=\operatorname{dim}_{\mathbf{Z} / \mathbf{p}}(X \times Y) \leq \operatorname{dim}_{\mathbf{Z} / \mathbf{p}} X+\operatorname{dim}_{\mathbf{Z} / \mathbf{p}} Y$ (see Theorem 22 of $\underline{\mathrm{Ku}}$ ), and, since $\operatorname{dim}_{\mathbf{Z} / \mathbf{p}} T \leq \operatorname{dim}_{F} T+1$ for any metrizable space $T$ and any $\mathbf{p}$-group $F$ (see Section 4 of $[\overline{\mathrm{Ku}}]$ or Theorem B of $\left[\mathrm{Dy}_{1} \mid\right.$ ), we are done. If $G * H$ is divisible by $\mathbf{p}$, then $\operatorname{dim}_{G * H}(X \times Y)=\operatorname{dim}_{\mathbf{Z} / \mathbf{p}^{\infty}}(X \times Y) \leq \operatorname{dim}_{\mathbf{Z} / \mathbf{p}^{\infty}} X+\operatorname{dim}_{\mathbf{Z} / \mathbf{p}^{\infty}} Y+1$ (see

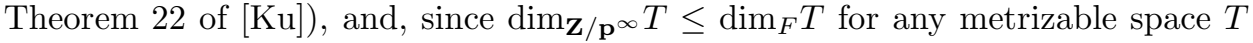

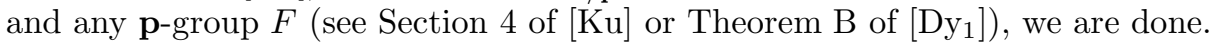

Now, let us prove that $\operatorname{dim}_{G \otimes H}(X \times Y) \leq m+n$. As in Section 5 of $[\mathrm{Ku}$, it suffices to show that $\operatorname{dim}_{H^{k}(Y / T ; G \otimes H)} X \leq m+n-k$ for all closed subsets $T$ of $Y$ 
and all $k$ such that $H^{k}(Y / T ; G \otimes H) \neq 0$. Since $\operatorname{dim}_{G \otimes H} Y \leq n$ (see Corollary 3.1 of $\left(\overline{\mathrm{Dy}_{1}}\right), H^{k}(Y / T ; G \otimes H) \neq 0$ implies $k \leq n$. By exactness of

$$
0 \rightarrow H^{k}(Y / T ; \mathbf{Z}) \otimes G \otimes H \rightarrow H^{k}(Y / T ; G \otimes H) \rightarrow H^{k+1}(Y / T ; \mathbf{Z}) *(G \otimes H) \rightarrow 0,
$$

and in view of

$$
\operatorname{dim}_{H^{k}(Y / T ; \mathbf{Z}) \otimes G \otimes H} X \leq m \leq m+n-k
$$

if $k \leq n$ (see Corollary 3.1 of $\left[\mathrm{Dy}_{1}\right]$ ), it suffices to show that

$$
\operatorname{dim}_{H^{k+1}(Y / T ; \mathbf{Z}) *(G \otimes H)} X \leq m+n-k
$$

if $k \leq n$. Let $F_{\mathbf{p}}$ be the p-torsion of $F=H^{k+1}(Y / T ; \mathbf{Z}) *(G \otimes H)$ for some prime $\mathbf{p}$. If $G$ is not divisible by $\mathbf{p}$, then either its $\mathbf{p}$-torsion is not divisible by $\mathbf{p}$ or $G$ / Tor $G$ is not divisible by $\mathbf{p}$. In either case $m+n-k \geq m=\operatorname{dim}_{G} X \geq \operatorname{dim}_{\mathbf{Z} / \mathbf{p}} X \geq \operatorname{dim}_{F_{\mathbf{p}}} X$. Thus, it remains to consider the case $G=\mathbf{p} \cdot G$.

Case 1: $F_{\mathbf{p}}$ is not divisible by $\mathbf{p}$. Since $G$ is divisible by $\mathbf{p}, H$ cannot be divisible by $\mathbf{p}$ as the $\mathbf{p}$-torsion of $G \otimes H$ would be trivial. Thus, the $\mathbf{p}$-torsion of $H^{k+1}(Y / T ; \mathbf{Z})$ is not divisible by $\mathbf{p}$. Hence $H^{k+1}(Y / T ; \mathbf{Z}) \otimes H \neq 0$, which implies $H^{k+1}(Y / T ; H) \neq$ 0 . The consequence is that $k<\operatorname{dim}_{H} Y=n$. If p-Tor $G=0$, then $\mathbf{p}-\operatorname{Tor}(G \otimes H)=$ 0 , and $F_{\mathbf{p}}=0$. Thus, p-Tor $G \neq 0$ and $m+n-k \geq m+1=\operatorname{dim}_{G} X+1 \geq$ $\operatorname{dim}_{\mathbf{Z} / \mathbf{p}^{\infty}} X+1 \geq \operatorname{dim}_{F_{\mathbf{p}}} X$.

Case 2: $F_{\mathbf{p}} \neq 0$ is divisible by $\mathbf{p}$. In this case both groups $H^{k+1}(Y / T ; \mathbf{Z})$ and $G \otimes H$ have non-trivial p-torsions which are divisible by $\mathbf{p}$. Since $G=\mathbf{p} \cdot G$, its p-torsion must be non-trivial and $m+n-k \geq m=\operatorname{dim}_{G} X \geq \operatorname{dim}_{\mathbf{Z} / \mathbf{p}^{\infty}} X=$ $\operatorname{dim}_{F_{\mathbf{p}}} X$.

Theorem 5.3. Suppose $X$ is a metrizable space, and $Y$ is a $\sigma$-compact metrizable space. Then

$$
\operatorname{dim}_{\mathbf{R}}(X \times Y) \leq \operatorname{dim}_{\mathbf{R}} X+\operatorname{dim}_{\mathbf{R}} Y
$$

for any ring $\mathbf{R}$ with unity, and

$$
\operatorname{dim}_{G}(X \times Y) \leq \operatorname{dim}_{G} X+\operatorname{dim}_{G} Y+1
$$

for any abelian group $G$.

Proof. If $\mathbf{R}$ is a ring with unity 1 , then $\mathbf{R}$ is a retract of $\mathbf{R} \otimes \mathbf{z} \mathbf{R}$. Indeed, the homomorphism $m: \mathbf{R} \otimes \mathbf{z} \mathbf{R} \rightarrow \mathbf{R}$ induced by the multiplication $\mathbf{R} \times \mathbf{R} \rightarrow \mathbf{R}$ is a left inverse of $i d \otimes 1: \mathbf{R} \rightarrow \mathbf{R} \otimes \mathbf{z} \mathbf{R}$. Thus,

$$
\operatorname{dim}_{\mathbf{R}}(X \times Y) \leq \operatorname{dim}_{\mathbf{R}} X+\operatorname{dim}_{\mathbf{R}} Y .
$$

Let $G$ be an abelian group. Since

$$
\operatorname{dim}_{G} Z=\max \left(\operatorname{dim}_{G} / \text { Tor } G, \operatorname{dim}_{\text {Tor } G} Z\right)
$$

for any metrizable space $Z$ (see $\left[\mathrm{Dy}_{1}\right)$ ), it suffices to prove

$$
\operatorname{dim}_{G}(X \times Y) \leq \operatorname{dim}_{G} X+\operatorname{dim}_{G} Y+1
$$

first for torsion groups, and then for torsion-free groups.

Since $\mathbf{Z} / \mathbf{p}$ is a ring with unity, the case $G=\mathbf{Z} / \mathbf{p}$ is taken care of. If $G=\mathbf{Z} / \mathbf{p}^{\infty}$ for some prime $\mathbf{p}$, then $G * G=G$, so $\operatorname{dim}_{G}(X \times Y) \leq \operatorname{dim}_{G} X+\operatorname{dim}_{G} Y+1$ by Theorem 5.2. By $\left[\mathrm{Dy}_{1}\right]$,

$$
\operatorname{dim}_{G}(X \times Y) \leq \operatorname{dim}_{G} X+\operatorname{dim}_{G} Y+1
$$

for any torsion group $G$. 
Consider a torsion-free abelian group $G$. Let $\mathcal{L}=\{\mathbf{p} \mid \mathbf{p} G \neq G\}$. By $\left[\mathrm{Dy}_{1}\right.$,

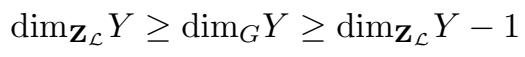

for any metrizable space $X$. Since $G \otimes \mathbf{Z}_{\mathcal{L}}=G$, by applying Theorem 5.2 to $H=\mathbf{Z}_{\mathcal{L}}$ one gets

$$
\operatorname{dim}_{G}(X \times Y) \leq \operatorname{dim}_{G} X+\operatorname{dim}_{\mathbf{z}_{\mathcal{L}}} Y \leq \operatorname{dim}_{G} X+\operatorname{dim}_{G} Y+1 .
$$

Using Theorems 5.2-5.3, we can prove analogs of the Menger Theorem in extension theory (5.4-5.6):

Theorem 5.4. Suppose $X$ is a metrizable space, and $Y$ is a $\sigma$-compact metrizable space. If $K \in A E(X)$ and $L \in A E(Y)$ are connected $C W$ complexes, then

$$
S P^{\infty}(K \wedge L) \in A E(X \times Y) .
$$

Proof. We may assume $Y$ is compact. Choose points $k \in K^{0}, l \in L^{0}$, and recall that the smash product $K \wedge L$ of $K$ and $L$ is $K \times L / K \vee L$, where $K \vee L=K \times l \cup k \times L$.

By Theorem 1.28 and Lemma 4 in [Ku] (p.34), we need to prove that

$$
\operatorname{dim}_{H_{q}(K \wedge L ; \mathbf{Z})}(X \times Y) \leq q
$$

for all $q$. By the Kunneth formula ([Sp], p.235), there is an exact sequence

$$
\begin{aligned}
0 \rightarrow[H(K, k ; \mathbf{Z}) \otimes H(L, l ; \mathbf{Z})]_{q} & \rightarrow H_{q}((K, k) \times(L, l) ; \mathbf{Z}) \\
& \rightarrow[H(K, k ; \mathbf{Z}) * H(L, l ; \mathbf{Z})]_{q-1} \rightarrow 0
\end{aligned}
$$

for all $q$. Now, by Theorem 3.4 of $\overline{\mathrm{Dy}_{1}}$,

$$
\operatorname{dim}_{H_{n}(K, k ; \mathbf{Z})} X \leq n
$$

and

$$
\operatorname{dim}_{H_{n}(L, l ; \mathbf{Z})} Y \leq n
$$

for all $n$. By Theorem 5.2,

$$
\operatorname{dim}_{[H(K, k ; \mathbf{Z}) \otimes H(L, l ; \mathbf{Z})]_{q}}(X \times Y) \leq q
$$

and

$$
\operatorname{dim}_{[H(K, k ; \mathbf{Z}) * H(L, l ; \mathbf{Z})]_{q-1}}(X \times Y) \leq q
$$

for all $q$. Finally (see Section 4 of $[\mathrm{Ku}]$ or $\left[\overline{\mathrm{Dy}_{1}}\right]$ ), $\operatorname{dim}_{H_{q}(K \wedge L ; \mathbf{Z})}(X \times Y) \leq q$ for all $q$.

Theorem 5.5. Suppose $X$ is a metrizable space of finite dimension, and $Y$ is a $\sigma$-compact metrizable space of finite dimension. If $K \in A E(X)$ and $L \in A E(Y)$ are connected $C W$ complexes, then

$$
K \wedge L \in A E(X \times Y) .
$$

Proof. Since both $K$ and $L$ are connected, we may assume that their 0-skeleta consist of single points $k$ and $l$. Now, the smash product $K \wedge L$ has no 1-skeleton, and is simply connected. By Theorem 5.4 and Theorem 1.28, $K \wedge L \in A E(X \times Y)$. 
Theorem 5.6. Suppose $X$ and $Y$ are compacta of finite dimension. If $K \in A E(X)$ and $L \in A E(Y)$ are $C W$ complexes, then

$$
\begin{aligned}
& K \wedge L \in A E(X \times Y), \\
& K \wedge L \in A E(X \wedge Y),
\end{aligned}
$$

and

$$
K * L \in A E(X * Y) .
$$

Proof. First, let us prove $K \wedge L \in A E(X \times Y)$. If both $K$ and $L$ are connected, this follows from Theorem 5.5. Assume $K$ is not connected. In this case $S^{0} \in A E(X)$, and any CW complex is an absolute extensor of $X$ (see [Dra4 $)$. Without loss of generality we may assume that $K$ is a simplicial complex with the weak topology. Since $Y \in S^{k} \wedge L$ and $Y \in I^{k} \wedge L$ for all $k \geq 0$ (see Dra5), one gets $Y \in A E(K \wedge L)$ by [Ko] $(K \wedge L$ is a union of absolute extensors of $Y$ with each finite intersection being an absolute extensor of $Y$ ). Suppose $f: A \rightarrow K \wedge L$ is continuous, and $A$ is a closed subset of $X \times Y$. Given $x \in X$, one can extend $f$ to $f_{s}: A \cup V_{s} \times Y \rightarrow K \wedge L$ for some open-closed neighborhood $V_{s}$ of $x$ (extend first $f$ over $x \times Y$ and then over its neighborhood). Now, it is clear that $f$ extends over $X \times Y$.

Since $X \wedge Y=X \times Y / X \vee Y$, we get $K \wedge L \in A E(X \wedge Y)$ and $\Sigma(K \wedge L) \in$ $A E(\Sigma(X \wedge Y))$ (see $\overline{D r a 5}$, Proposition 6). Notice that $\Sigma(K \wedge L) \simeq K * L$. Since $K * L \in A E(X), K * L \in A E(Y)$ and $K * L \in A E(X * Y-X \oplus Y)$, we get $K * L \in A E(X * Y)$ (see Dra5, Proposition 3).

Corollary 5.7. Suppose $X$ and $Y$ are separable metrizable spaces of finite dimension. If $K \in A E(X)$ and $L \in A E(Y)$ are connected $C W$ complexes, and $L$ is homotopy dominated by a finite $C W$ complex, then

$$
K \wedge L \in A E(X \times Y) .
$$

Proof. By Corollary 2.5 of $\mathrm{Dy}_{2}$ there is a compact space $Y^{\prime}$ containing $Y$ such that $L \in A E\left(Y^{\prime}\right)$. By Theorem 5.5, $K \wedge L \in A E\left(X \times Y^{\prime}\right)$. Now, use Proposition 1.1 of $[\overline{\mathrm{Dy}} 2]$.

Theorem 5.8. Suppose $W$ is a separable metrizable space of finite dimension, $Y$ is a $\sigma$-compact metrizable space of finite dimension, and $G_{1}, \ldots, G_{m}$ are countable abelian groups. Then, for any $k$ so that $0<k<\operatorname{dim}_{G_{i}} W-\operatorname{dim}_{G_{i}} Y$ for all $i \leq m$, there is a closed subset $T$ of $W$ such that

$$
\operatorname{dim}_{G_{i}} T=\operatorname{dim}_{G_{i}} W-k
$$

and

$$
\operatorname{dim}_{G_{i}}(T \times Y)=\operatorname{dim}_{G_{i}}(W \times Y)-k
$$

for all $i \leq m$.

Proof. It suffices to consider the case $k=1$. First, assume $Y$ is compact. Let $\left\{V_{i}\right\}$ be a countable basis of $Y$. Suppose $X$ is a metrizable space. Notice that $\operatorname{dim}_{G}(X \times Y) \leq n$ is equivalent to $H^{n+1-j}\left(X, X-U ; H^{j}\left(Y, Y-V_{i} ; G\right)\right)=0$ for all $i, j$ and all open subsets $U$ of $X$, which is equivalent to $\operatorname{dim}_{H^{j}\left(Y, Y-V_{i} ; G\right)} X \leq n-j$ for all $i, j$ (see Section 5 of $[\mathrm{Ku}$ ). Given $j \leq \operatorname{dim} Y$, and given $n \leq m$, let $G(n, j)$ be the direct sum of all the groups $H^{j}\left(Y, Y-V_{i} ; G_{n}\right), i \geq 1$. Choose a closed subset $T$ of $W$ such that $\operatorname{dim} T=\operatorname{dim}_{\mathbf{Z}} T=\operatorname{dim}_{\mathbf{Z}} W-1=\operatorname{dim} W-1, \operatorname{dim}_{G_{i}} T=$ $\operatorname{dim}_{G_{i}} W-1$ for $i \leq m$, and $\operatorname{dim}_{G(n, j)} T=\operatorname{dim}_{G(n, j)} W-1$ if $\operatorname{dim}_{G(n, j)} W>1$. 
Clearly, $\operatorname{dim}_{G_{i}}(T \times Y) \leq \operatorname{dim}_{G_{i}}(W \times Y)-1$ all $i \leq n$. Suppose $\operatorname{dim}_{G_{s}}(W \times Y)=d$ for some $s \leq n$. That means there is $i, j$ such that $\operatorname{dim}_{H^{j}\left(Y, Y-V_{i} ; G_{s}\right)} W=d-j$. Now, since $d-j>1\left(H^{j}\left(Y, Y-V_{i} ; G_{s}\right) \neq 0\right.$ means $j \leq \operatorname{dim}_{G_{s}} Y<\operatorname{dim}_{G_{s}} W-1 \leq$ $\left.\operatorname{dim}_{G_{s}}(W \times Y)-1=d-1\right)$, then $\operatorname{dim}_{G(s, j)} T=d-j-1$, and there is $t$ such that $\operatorname{dim}_{H^{j}\left(Y, Y-V_{t} ; G_{s}\right)} T=d-j-1$, which implies $\operatorname{dim}_{G_{s}}(T \times Y)=d-1$.

If $Y$ is not compact, we express $Y$ as the union of an increasing sequence $Y_{n}$ of its compact subsets. Since $\operatorname{dim}_{G} Y=\max \left\{\operatorname{dim}_{G} Y_{n} \mid n \geq 1\right\}$ and $\operatorname{dim}_{G}(W \times Y)=$ $\max \left\{\operatorname{dim}_{G}\left(W \times Y_{n}\right) \mid n \geq 1\right\}$ for all groups $G$, there is $n$ such that $\operatorname{dim}_{G_{i}} Y=$ $\operatorname{dim}_{G_{i}} Y_{n}$ and $\operatorname{dim}_{G_{i}}(W \times Y)=\operatorname{dim}_{G_{i}}\left(W \times Y_{n}\right)$ for $i \leq m$. Choose $T$ for the space $Y_{n}$

Corollary 5.9. Suppose $W$ is a separable metrizable space of finite dimension, and $Y$ is a $\sigma$-compact metrizable space of finite dimension. Then, for any $k, 0<k<$ $\operatorname{dim} W-\operatorname{dim} Y$, there is a closed subset $T$ of $W$ such that

$$
\operatorname{dim} T=\operatorname{dim} W-k
$$

and

$$
\operatorname{dim}(T \times Y)=\operatorname{dim}(W \times Y)-k
$$

Dranishnikov, Repovš and Ščepin $\overline{D-R-S_{2}}$ found the only example so far where the cartesian product $W \times Y$ of a 2-dimensional subset $W$ of $R^{3}$ and a 1-dimensional continuum $Y$ is 2-dimensional. We are uncertain if this pathology is restricted to low dimensions only.

Problem 5.10. Suppose $n>1$. Is there a separable metrizable space $W$ of dimension $n+1$ such that $\operatorname{dim}(W \times Y)=\operatorname{dim} W$ for some compactum $Y$ of dimension $n$ ?

Notice that if $\operatorname{dim} Y=n$ and $\operatorname{dim}(W \times Y)=\operatorname{dim} W$ for some separable space of dimension greater than $n$, then Corollary 5.9 implies the existence of $T$ closed in $W$ such that $n+1=\operatorname{dim} T=\operatorname{dim}(T \times Y)$. Also, notice that if $\operatorname{dim} Y=\operatorname{dim} W>0$, then $\operatorname{dim}(W \times Y) \geq \operatorname{dim} W+1$ (see [Ku], Corollary on p.38).

\section{REFERENCES}

[A] P.S.Alexandroff, Dimensionstheorie, Ein Beitrag zur Geometrie der abgeschlossenen Mengen, Math. Ann. 106 (1932), 161-238.

[Bor] K.Borsuk, Un theoreme sur la prolongements des transformations, Fund. Math. 29 (1937), 161-166.

[Ca] R. Cauty, Sur les sous-espaces des complexes simpliciaux, Bull. Soc. Math. France 100 (1972), 129-155. MR 48:5023

[Co] H. Cohen, A cohomological definition of dimension for locally compact Hausdorff spaces, Duke Math. J. 21 (1954), 209-224. MR 16:609b

[D-M] J.Dydak and J.Mogilski, Universal cell-like maps, Proceedings of AMS 122 (1994), 943-948. MR 95a:55003

[Dra 1 A.N.Dranishnikov, Homological dimension theory, Russian Math. Surveys 43(4) (1988), 11-63. MR 90e:55003

[Dra 2 A.N.Dranishnikov, On the mapping intersection problem, Pacific Journal of Mathematics 173 No.2 (1996), 403-412. MR 97e:54030]

[Dra 3 A.N.Dranishnikov, Extension of maps into $C W$ complexes, Math. USSR Sbornik 74 (1993), 47-56. MR 93a:55002 
[Dra 4 A.N.Dranishnikov, Eilenberg-Borsuk theorem for maps into arbitrary complexes, Math. Sbornik 185 (1994), 81-90. MR 95j:54028

[Dra5] A.N.Dranishnikov, On intersection of compacta in Euclidean space II, Proceedings of AMS 113 (1991), 1149-1154. MR 92c:54015

[Dra6] A.N.Dranishnikov, Cohomological dimension is not preserved by Stone-Čech compactification, Comptes Rendus Bulgarian Acad. of Sci. 41 (1988), no. 12, 9-10 (Russian). MR 90e:55002

[Dra7] A.N.Dranishnikov, On the dimensions of the product, the union and the intersection of two spaces, preprint, 1995.

[D-R] A.Dranishnikov and D.Repovš, The Urysohn-Menger Sum Formula: An extension of the Dydak-Walsh theorem to dimension one, J.Austral. Math. Soc., Ser.A 59 (1995), 273-282. MR 96g:55003

[D-R-S 1 A.Dranishnikov, D.Repovš and E.Ščepin, On the failure of the Urysohn-Menger sum formula for cohomological dimension, Proc.Amer.Math.Soc. 120 (1994), 1267-1270. MR 94f:55001

[D-R-S $\left.{ }_{2}\right]$ A.N. Dranishnikov, D. Repovš and E.Ščepin, Dimension of products with continua, Topology Proceedings 18 (1993), 57-73. MR 96b:54054

[D-S] J.Dydak and J.Segal, Shape theory: An introduction, Lecture Notes in Math. 688, Springer Verlag, 1978, pp. 1-150. MR 80h:54020

[D-T] A.Dold and R.Thom, Quasifaserungen und Unendliche Symmetrische Produkte, Annals of Math. 67 (1958), 239-281. MR 20:3542

$\left[\mathrm{D}_{-} \mathrm{W}_{1}\right] \quad J . D y d a k$ and J.J.Walsh, Spaces without cohomological dimension preserving compactifications, Proceedings of the Amer.Math.Soc. 113 (1991), 1155-1162. MR 92c:54039

$\left[\mathrm{D}-\mathrm{W}_{2}\right]$ J.Dydak and J.J.Walsh, Aspects of cohomological dimension for principal ideal domains, preprint.

[Dy1] J.Dydak, Cohomological dimension and metrizable spaces, Transactions of the Amer.Math.Soc. 337 (1993), 219-234. MR 93g:55001

[Dy2] J.Dydak, Cohomological dimension and metrizable spaces II, Trans.Amer.Math.Soc. 348 (1996), 1647-1661. MR 96h:55001

[Dy 3 J.Dydak, Union theorem for cohomological dimension: A simple counterexample, Proceedings of AMS 121 (1994), 295-297. MR 94g:55001

[Ei] S.Eilenberg, Un theoreme de la dualite, Fund. Math. 26 (1936), 280-282.

[En] R.Engelking, Dimension Theory, Math. Library, North-Holland, 1978. MR 58:2753b

[Fu] L.Fuchs, Infinite abelian groups, Academic Press, New York and London, 1970. MR 41:333

$[\mathrm{Hu}]$ S.T.Hu, Theory of retracts, Wayne State University Press, 1965, pp. 1-234. MR 31:6202

[Kob] N.Koblitz, p-Adic Numbers, $p$-Adic Analysis, and Zeta-functions, Springer-Verlag, New York, Heidelberg, Berlin, 1977. MR 57:5964

[Ko] Y. Kodama, Note on an absolute neighborhood extensor for metric spaces, Journal of the Mathematical Society of Japan 8 (1956), 206-215. MR 18:406c

[Ku] V.I. Kuzminov, Homological dimension theory, Russian Math. Surveys 23 (1968), no. 5, 1-45. MR 39:2158

[M-S] S.Mardesic and J.Segal, Shape theory, North-Holland Publ.Co., Amsterdam, 1982. MR 84b:55020

[Mas] W.Massey, Homology and Cohomology Theory, Marcel Dekker, New York, Basel, 1978. MR 58:7594

$\left[\mathrm{Ol}_{1}\right] \quad$ W.Olszewski, Completion theorem for cohomological dimensions, Proceedings of AMS 123 (1995), 2261-2264. MR 95k:54064

$\left[\mathrm{Ol}_{2}\right]$ W.Olszewski, Universal separable metrizable spaces for cohomological dimension, Topology and its Appl. 61 (1995), 293-299. MR 95m:54013

[Ru] L.R.Rubin, Characterizing cohomological dimension: The cohomological dimension of $A \cup B$, Topology and its Appl. 40 (1991), 233-263. MR 92g:55002

[R-S] L.R.Rubin and P.J.Schapiro, Cell-like maps onto non-compact spaces of finite cohomological dimension, Topology and its Appl. 27 (1987), 221-244. [MR 89h:55002

[Sp] E.Spanier, Algebraic topology, McGraw-Hill, New York, 1966. MR 35:1007]

[Su] D.Sullivan, Geometric Topology, Part I: Localization, Periodicity, and Galois Symmetry, M.I.T. Press, 1970. MR 58:13006a 
[Wa] J.J.Walsh, Dimension, cohomological dimension, and cell-like mappings, Lecture Notes in Math. 870, 1981, pp. 105-118. MR 83a:57021

[We] J.West, Open problems in infinite dimensional topology, in Open Problems in Topology, North-Holland, 1990, pp. 523-597. MR 92c:54001

[Wh] G.W.Whitehead, Elements of homotopy theory, Springer-Verlag, 1978. MR 80b:55001

[Z] M.Zarichnyi, Universal spaces for cohomological dimension (to appear).

Department of Mathematics, University of Florida, Gainesville, Florida 32611

E-mail address: dranish@math.ufl.edu

Department of Mathematics, University of Tennessee, Knoxville, Tennessee 37996

E-mail address: dydak@math.utk.edu 